\title{
A Chiral [3]Rotaxane Comprising Achiral Bis-macrocyclic and Dumbbell-Shaped Components
}

Takaaki Morise, Atsuya Muranaka,* Hayato Ban, Mei Harada, Masaya Naito, Kazuyuki Yoshida, Nagao Kobayashi, Masanobu Uchiyama, and Yuji Tokunaga*

\section{Supporting Information}

TOC

1. Experimental. 1

2. Figure S1. ${ }^{1} \mathrm{H}$ NMR spectra of the [3] rotaxane 1.

3. Figure S2. ${ }^{13} \mathrm{C}$ NMR spectra of the [3] rotaxane $\mathbf{1 .} 7$

4. Figure S3. COSY spectrum of the [3]rotaxane $1 . \quad 10$

5. Figure S4. ROESY spectrum of the [3] rotaxane 1. 12

6. Figure S5. HSQC spectrum of the [3]rotaxane 1. 14

7. Figure S6. HMBC spectrum of the [3] rotaxane 1. 16

8. Figure S7. Mass spectrum of the [3] rotaxane 1. 19

9. Table S1 \& Figure S8. Crystal structure and data of the [3] rotaxane 1. 20

10. Figure S9. Chiral HPLC chromatogram of the [3] rotaxane 1. 23

11. Figure S10. Cartoon representation of the absolute configurations of the enantiomers of the [3] rotaxane 1.

12. Computational details. $\quad 24$

13. Table S2. Selected TD-DFT data for $(R)-\mathbf{1}^{\prime}$. 24

14. Figure S11. Selected molecular orbitals of $(R)-\mathbf{1}^{\prime}$. 24

15. Cartesian coordinates and energies of $(R)-\mathbf{1}^{\prime}$. 25

$\begin{array}{ll}\text { 16. References. } & 29\end{array}$

17. Figure S12. ${ }^{1} \mathrm{H}$ NMR spectrum of compound 3 . 30

18. Figure S13. ${ }^{1} \mathrm{H}$ and ${ }^{13} \mathrm{C}$ NMR spectra of compound 4.

19. Figure $\mathbf{S 1 4}{ }^{1} \mathrm{H}$ and ${ }^{13} \mathrm{C}$ NMR spectra of compound 5 . 32

20. Figure S15. ${ }^{1} \mathrm{H}$ and ${ }^{13} \mathrm{C}$ NMR spectra of compound 6.

21. Figure S16. ${ }^{1} \mathrm{H}$ and ${ }^{13} \mathrm{C}$ NMR spectra of the [3]rotaxane $\mathbf{1 .}$ 


\section{Experimental}

\section{Materials and General Methods}

The calix-bis-crown ether $\mathbf{1}^{\mathrm{S} 1}$ and the amine $\mathbf{3}^{\mathrm{S} 2}$ were prepared according to literature procedures. All solvents and commercially available chemicals were used as received. ${ }^{1} \mathrm{H}$ and ${ }^{13} \mathrm{C}$ NMR spectra were recorded using JEOL ECX-500II and ECA-600II spectrometers, with tetramethylsilane (TMS) as the internal standard. Mass spectra were recorded using JEOL JMS-700T (FAB) and Bruker micrOTOF (MALDI) spectrometers. Infrared spectra were recorded using a Shimadzu FTIR-8600PC spectrometer. CD and UV spectra were recorded using JASCO J-820 and HITACHI U-3900H spectrophotometers. All reactions were performed under a positive atmosphere of dry $\mathrm{N}_{2}$. All solvents were removed through rotary evaporation under reduced pressure. Silica gel column chromatography was performed using Kanto Chemical silica gel 60N. Thin layer chromatography was performed using Merck Kieselgel 60PF 254 . HPLC was performed using a Shimadzu LC-20AT apparatus and a SDD-M20A detector, with DAICEL CHIRALPAK IA3 chiral column $(0.40 \times 25 \mathrm{~cm})$ for analysis, and DAICEL CHIRALPAK IA chiral column $(1.0 \times 25 \mathrm{~cm})$ for separation.

\section{X-ray Diffractometry (XRD)}

Crystals of the [3]rotaxane 1 were subjected to XRD analysis using a Rigaku Saturn-70 diffractometer and graphite-monochromated synchrotron radiation $(\lambda=0.81042 \AA)$. The structure of 1 was solved using direct methods and refined by applying the full-matrix least-squares method. In subsequent refinement, the function $\Sigma \omega\left(F_{\mathrm{o}}{ }^{2}-F_{\mathrm{c}}{ }^{2}{ }^{2}\right.$ was minimized, where $F_{\mathrm{o}}$ and $F_{\mathrm{c}}$ were the observed and calculated structure factor amplitudes. The positions of non-hydrogen atoms were determined from difference Fourier electron density maps and refined anisotropically. All calculations were performed using the CrystalStructure crystallographic software package; the illustration was generated in the ORTEP style. ${ }^{\mathrm{S} 3}$ Details of the structural determination of $\mathbf{1}$ are provided in Figures 3 and S8 and Table S1. CCDC 2047960 contains the supplementary crystallographic data for this paper.

\section{Ammonio Ester Salt 4}

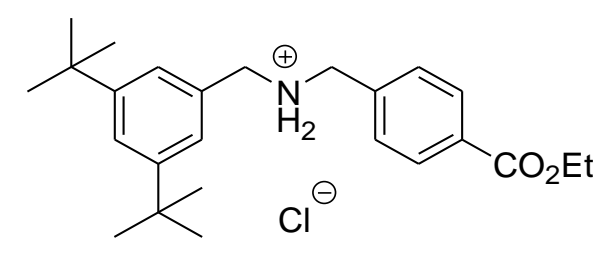

A suspension of the amine 3 (1.29 g, $5.88 \mathrm{mmol})$, 4-(ethoxycarbonyl)benzaldehyde (1.10 $\mathrm{g}, 6.17 \mathrm{mmol})$, and $\mathrm{Na}_{2} \mathrm{SO}_{4}(0.668 \mathrm{~g}, 4.70 \mathrm{mmol})$ in toluene $(15 \mathrm{~mL})$ was stirred overnight 
at room temperature. After filtration of the mixture, the filtrate was concentrated under reduced pressure. The residue was dissolved in $\mathrm{EtOH}(30 \mathrm{~mL})$ and then $\mathrm{NaBH}_{4}(0.222 \mathrm{~g}$, $5.88 \mathrm{mmol}$ ) was added to the solution cooled in a water bath. After stirring overnight at room temperature, dil. $\mathrm{HCl}$ (aq.) was added and then the mixture was stirred for $10 \mathrm{~min}$. The mixture was neutralized with dil. $\mathrm{Na}_{2} \mathrm{CO}_{3}$ (aq.) and then the EtOH was evaporated. The aqueous phase was extracted with AcOEt. The combined extracts were washed with sat. $\mathrm{NaCl}$ (aq.), dried $\left(\mathrm{Na}_{2} \mathrm{SO}_{4}\right)$, and concentrated. The residue was purified chromatographically $\left(\mathrm{SiO}_{2}\right.$; hexane/AcOEt, 7:1) to give the amine, which was dissolved in hexane $(20 \mathrm{~mL})$, and treated with $4 \mathrm{~N} \mathrm{HCl}$ in AcOEt $(1.47 \mathrm{~mL}, 5.88 \mathrm{mmol})$. Filtration of the precipitate afforded the ammonium salt $4(1.68 \mathrm{~g}, 68 \%)$ as a white powder. IR (KBr, $\left.v_{\max }, \mathrm{cm}^{-1}\right)$ : 3423, 2962, 2779, 2605, 1720, 1602, 1578, 1449, 1364, 1275, 1106, 1024, 869. ${ }^{1} \mathrm{H}$ NMR (500 MHz, $\left.\mathrm{CDCl}_{3}\right) \delta$ : 10.52 (br s, 2H), 7.99-8.05 (m, 2H), 7.55-7.61 (m, 2H), 7.41 (d, $J=1.5 \mathrm{~Hz}, 1 \mathrm{H}), 7.31$ (br s, 2H), 4.32 (q, $J=7.1 \mathrm{~Hz}, 2 \mathrm{H}), 3.91$ (br t, $J=4.6$ $\mathrm{Hz}, 2 \mathrm{H}), 3.87$ (br t, $J=4.9 \mathrm{~Hz}, 2 \mathrm{H}), 1.35$ (t, $J=7.1 \mathrm{~Hz}, 3 \mathrm{H}), 1.31$ (s, 18H). ${ }^{13} \mathrm{C} \mathrm{NMR}$ (125 MHz, $\left.\mathrm{CDCl}_{3}\right): \delta: 165.8,151.9,134.9,131.3,130.4,130.1,128.7,124.5,123.3,61.1$, 49.1, 47.7, 35.0, 31.4, 14.2. HRMS (FAB) calcd. for $\mathrm{C}_{25} \mathrm{H}_{36} \mathrm{NO}_{2}{ }^{+},[\mathrm{M}-\mathrm{Cl}]^{+} \mathrm{m} / z$ 382.2741, found 382.2725 .

\section{Amino Acid Salt 5}

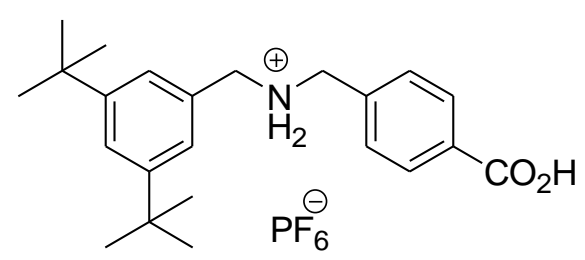

$10 \% \mathrm{NaOH}$ (aq.) (4.0 mL) was added to a solution of the ester $4(1.63 \mathrm{~g}, 3.90 \mathrm{mmol})$ in $\mathrm{MeOH}(20 \mathrm{~mL})$ and then the mixture was stirred for $4 \mathrm{~h}$ at $50{ }^{\circ} \mathrm{C}$. After evaporation of the $\mathrm{MeOH}, 10 \% \mathrm{HCl}$ (aq.) was added to the reaction mixture to $\mathrm{pH} 1$. The precipitate was collected by filtration to afford the corresponding ammonium chloride, which was suspended in acetone $(25 \mathrm{~mL})$ and water $(15 \mathrm{~mL}) . \mathrm{NH}_{4} \mathrm{PF}_{6}(1.91 \mathrm{~g}, 11.7 \mathrm{mmol})$ was added to the suspension and then the mixture was stirred for $1 \mathrm{~h}$ at room temperature. After evaporation of the acetone, the precipitate was filtered off and washed with water to give the ammonium salt $5(1.74 \mathrm{~g}, 89 \%)$ as a white powder. IR $\left(\mathrm{KBr}, v_{\max }, \mathrm{cm}^{-1}\right): 3426,2961$, 2676, 1700, 1618, 1604, 1583, 1479, 1463, 1429. 1403, 1364, 1322, 1287, 1253, 1211, 944, 858. ${ }^{1} \mathrm{H}$ NMR (500 MHz, DMSO- $\left.d_{6}\right) \delta:$ 7.95-8.03 (m, 2H), 7.57-7.64 (m, 2H), 7.41 (s, 1H), 7.30 (s, 2H), 4.26 (br s, 2H), 4.14 (br s, 2H), 1.28 (s, 18H). ${ }^{13} \mathrm{C} \mathrm{NMR} \mathrm{(125} \mathrm{MHz,}$ DMSO- $\left.d_{6}\right): \delta: 167.0,150.8,137.2,131.7,131.3,130.0,129.6,123.9,122.5,51.1,50.1$, 34.6, 31.2. HRMS (FAB) calcd. for $\mathrm{C}_{23} \mathrm{H}_{32} \mathrm{NO}_{2}{ }^{+},\left[\mathrm{M}-\mathrm{PF}_{6}\right]^{+} \mathrm{m} / z$ 354.2428, found 354.2385 . 


\section{[3]Rotaxane 1}

$\mathrm{Ph}_{2} \mathrm{CN}_{2}$ (126 mg, $0.650 \mathrm{mmol}$ ) was added to a solution of the ammonium salt 5 (177 $\mathrm{mg}$, $0.500 \mathrm{mmol})$ and the bis-crown ether $2(50.3 \mathrm{mg}, 0.0500 \mathrm{mmol})$ in $\mathrm{CHCl}_{3} / \mathrm{CH}_{3} \mathrm{CN}$ (1:1, $1.25 \mathrm{~mL}$ ) at room temperature. After stirring for $2 \mathrm{~h}$, the mixture was concentrated. The residue was purified chromatographically $\left(\mathrm{SiO}_{2}\right.$; toluene/acetone, 2:1) to give the [3] rotaxane 1 (80.0 mg, 69\%) as a white solid. IR (KBr, $\left.v_{\max }, \mathrm{cm}^{-1}\right): 3064,3032,2963$, 2906, 2871, 1723, 1601, 1580, 1457, 1363, 1355, 1270, 1215, 1093, 1022, 956, 846, 765, 703, 557. ${ }^{1} \mathrm{H} \mathrm{NMR}\left(600 \mathrm{MHz}, \mathrm{CDCl}_{3}\right) \delta: 8.29-8.33$ (m, 4H), 7.83-7.98 (m, 4H), 7.61$7.66(\mathrm{~m}, 4 \mathrm{H}), 7.28$ (br t, $J=7.7 \mathrm{~Hz}, 2 \mathrm{H}), 7.33$ (s, 4H), 7.28 (br t, $J=7.7 \mathrm{~Hz}, 2 \mathrm{H}), 7.12$ (s, 2H), 6.94-7.00 (m, 8H), 6.47-6.52 (m, 4H), 4.23-4.34 (m, 4H), 4.13-4.22 (m, 4H), 3.82$3.91(\mathrm{~m}, 4 \mathrm{H}), 3.49-3.81(\mathrm{~m}, 40 \mathrm{H}), 3.18-3.25(\mathrm{~m}, 4 \mathrm{H}), 3.02-3.08(\mathrm{~m}, 4 \mathrm{H}), 2.91-2.99$ (br $\mathrm{s}, 16 \mathrm{H}), 1.37$ (s, 36H). ${ }^{13} \mathrm{C} \mathrm{NMR}\left(150 \mathrm{MHz}, \mathrm{CDCl}_{3}\right) \delta: 164.8,156.2,156.1,151.6,140.0$, $136.1,133.55,133.52$, 133.38, 133.36, 131.6, 130.4, 130.2, 129.8, 128.7, 128.0, 127.1, 125.0, 124.1, 122.5, 122.3, 78.1, 71.3, 70.7, 70.3, 70.2, 70.0, 68.1, 66.4, 66.3, 52.8, 51.0, 38.0, 35.0, 31.5. HRMS (MALDI) calcd. for $\mathrm{C}_{128} \mathrm{H}_{160} \mathrm{~N}_{2} \mathrm{O}_{20}{ }^{2+}\left[\mathrm{M}-2 \mathrm{PF}_{6}\right]^{2+} \mathrm{m} / \mathrm{z}$ 1022.5777, found 1022.5776 .

\section{Dumbbell-Shaped Salt 6}

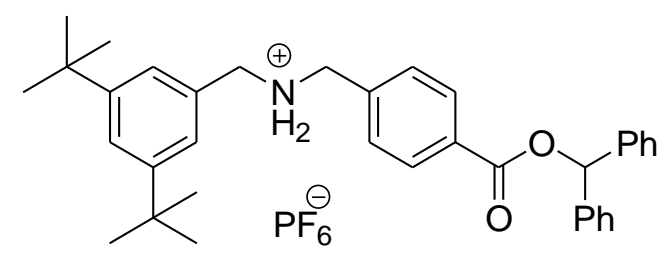

$\mathrm{Ph}_{2} \mathrm{CN}_{2}$ (30 mg, $0.16 \mathrm{mmol}$ ) was added to a solution of the ammonium salt 5 (50 $\mathrm{mg}$, $0.14 \mathrm{mmol})$ in $\mathrm{CHCl}_{3} / \mathrm{CH}_{3} \mathrm{CN}(1: 1,0.75 \mathrm{~mL})$ at room temperature. After stirring for $3 \mathrm{~h}$, the mixture was concentrated. The residue was washed with isopropyl ether to afford the dumbbell-shaped salt 6 (73 mg, 78\%) as a white solid. IR ( $\left.\mathrm{KBr}, v_{\max }, \mathrm{cm}^{-1}\right)$ : 3452, 2963, 2905, 2868, 1720, 1602, 1477, 1456, 1408, 1363, 1316, 1272, 1185, 1103, 859. ${ }^{1} \mathrm{H}$ NMR (500 MHz, $\left.\mathrm{CDCl}_{3}\right)$ \&: 8.05-8.16 (m, 2H), 7.25-7.49 (m, 13H), $7.17(\mathrm{~s}, 2 \mathrm{H}), 7.05$ (s, 1H), 6.87 (br s, 2H), 4.07-4.34 (m, 4H), 1.28 (s, 18H). $\left.{ }^{13} \mathrm{C} \mathrm{NMR} \mathrm{(150} \mathrm{MHz,} \mathrm{CDCl}_{3}\right)$ $\delta: 164.8,152.6,139.8,133.9,131.8,130.8,129.9,128.6,128.1,127.8,127.1,124.4$, 123.9, 78.0, 52.3, 50.5, 34.9, 31.2. HRMS (FAB) calcd. for $\mathrm{C}_{36} \mathrm{H}_{42} \mathrm{NO}_{2}{ }^{+}\left[\mathrm{M}-\mathrm{PF}_{6}\right]^{+} \mathrm{m} / \mathrm{z}$ 520.3210 , found 520.3208 . 

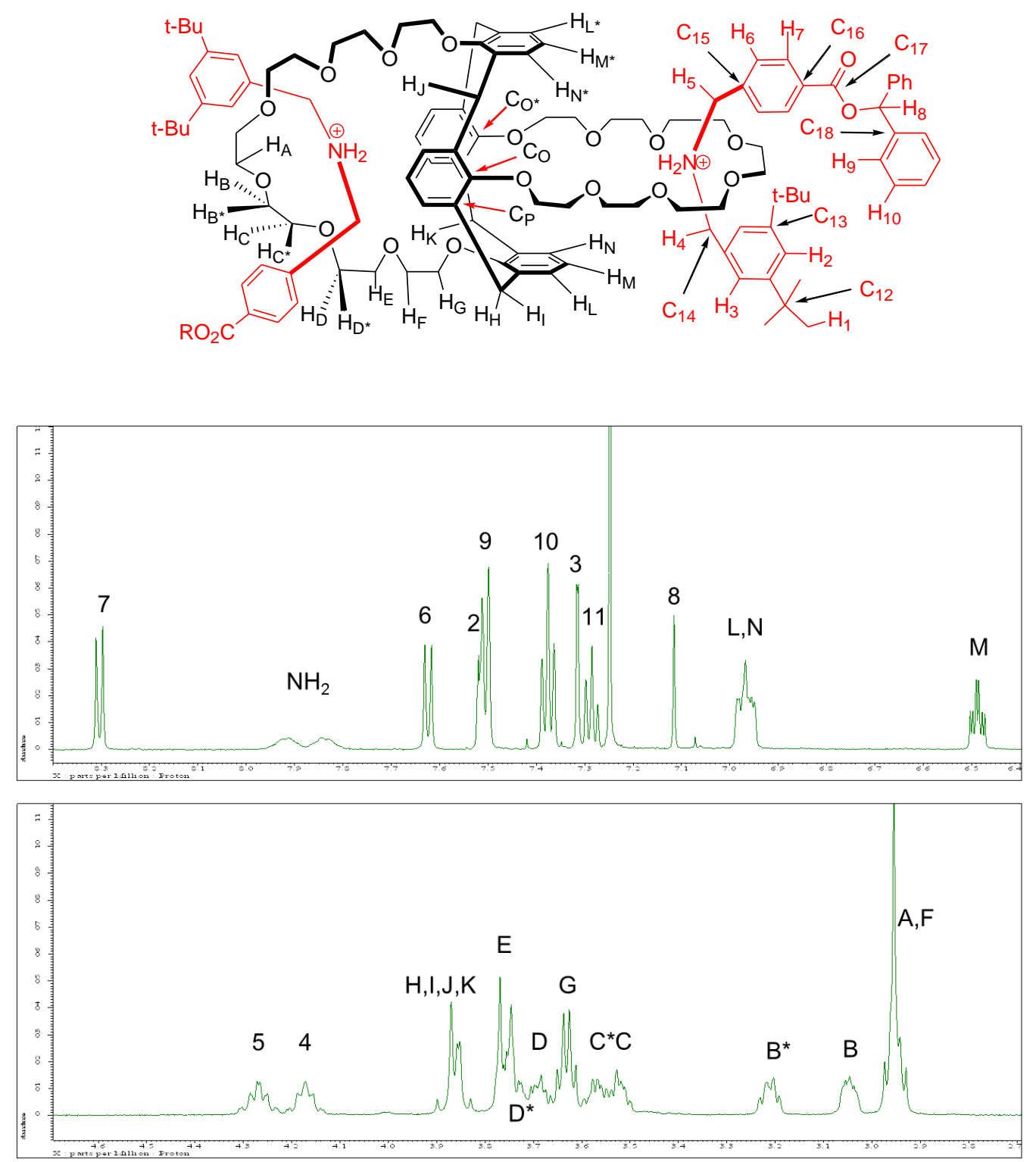

Figure S1a. ${ }^{1} \mathrm{H}$ NMR spectra $\left(600 \mathrm{MHz}, \mathrm{CDCl}_{3}, 22{ }^{\circ} \mathrm{C}\right)$ of the [3] rotaxane $\mathbf{1}$. 


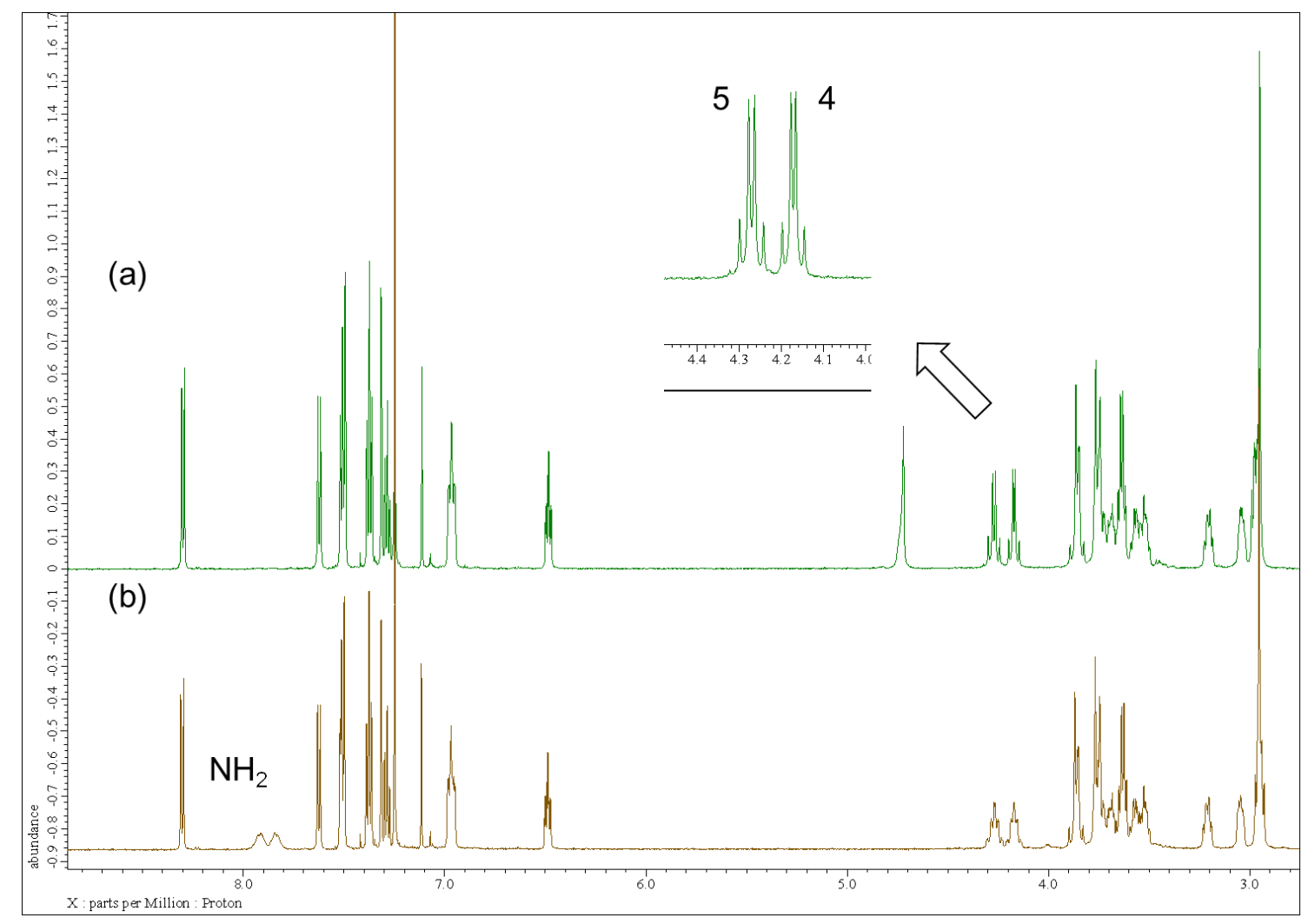

Figure S1b. ${ }^{1} \mathrm{H}$ NMR spectra $\left(600 \mathrm{MHz}, \mathrm{CDCl}_{3}, 22{ }^{\circ} \mathrm{C}\right)$ of the [3] rotaxane 1 (a) in the presence of $\mathrm{D}_{2} \mathrm{O}$ and (b) in the absence of $\mathrm{D}_{2} \mathrm{O}$. 

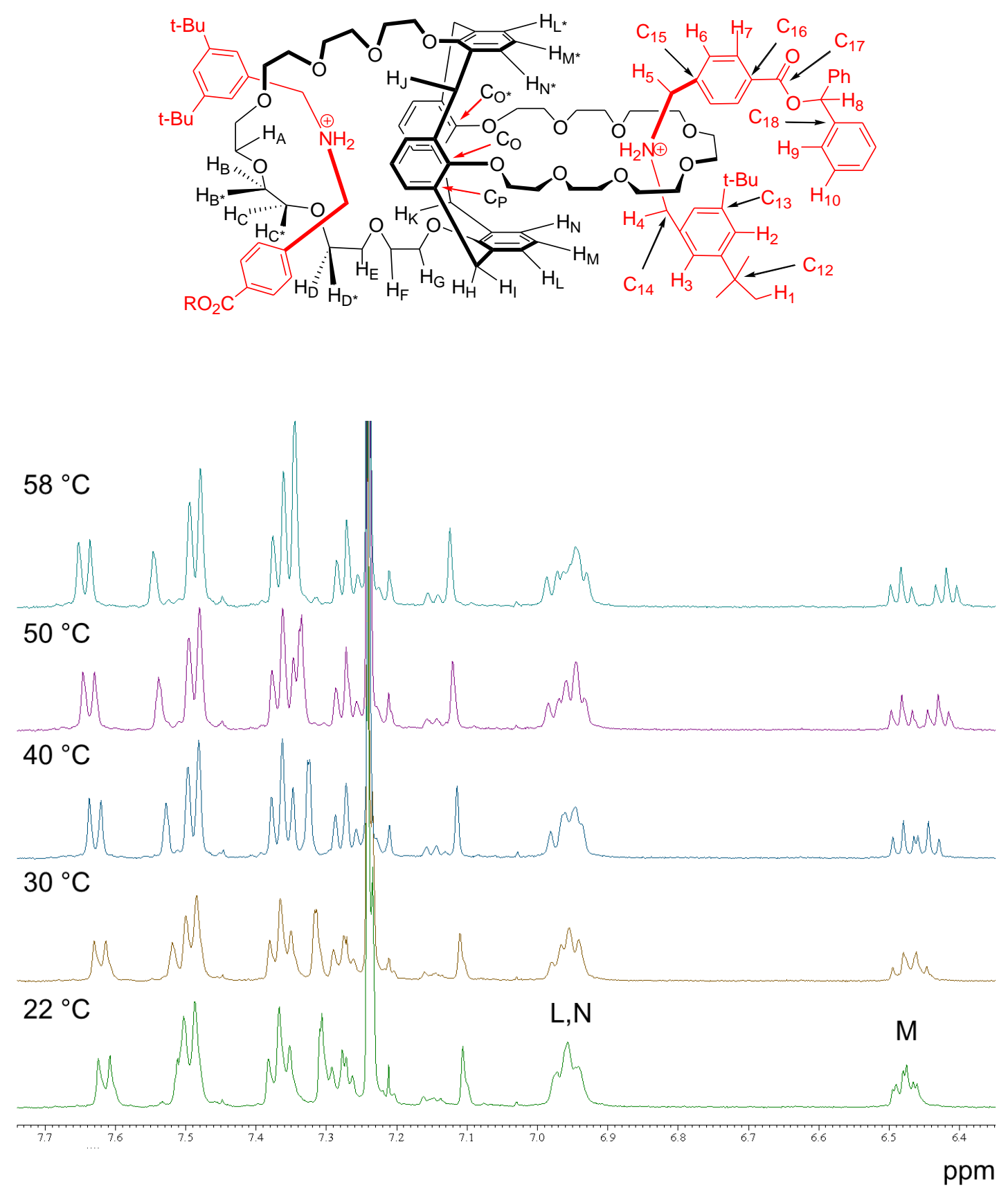

Figure S1c. VT NMR spectra $\left(600 \mathrm{MHz}, \mathrm{CDCl}_{3}\right)$ of the [3] rotaxane 1. 

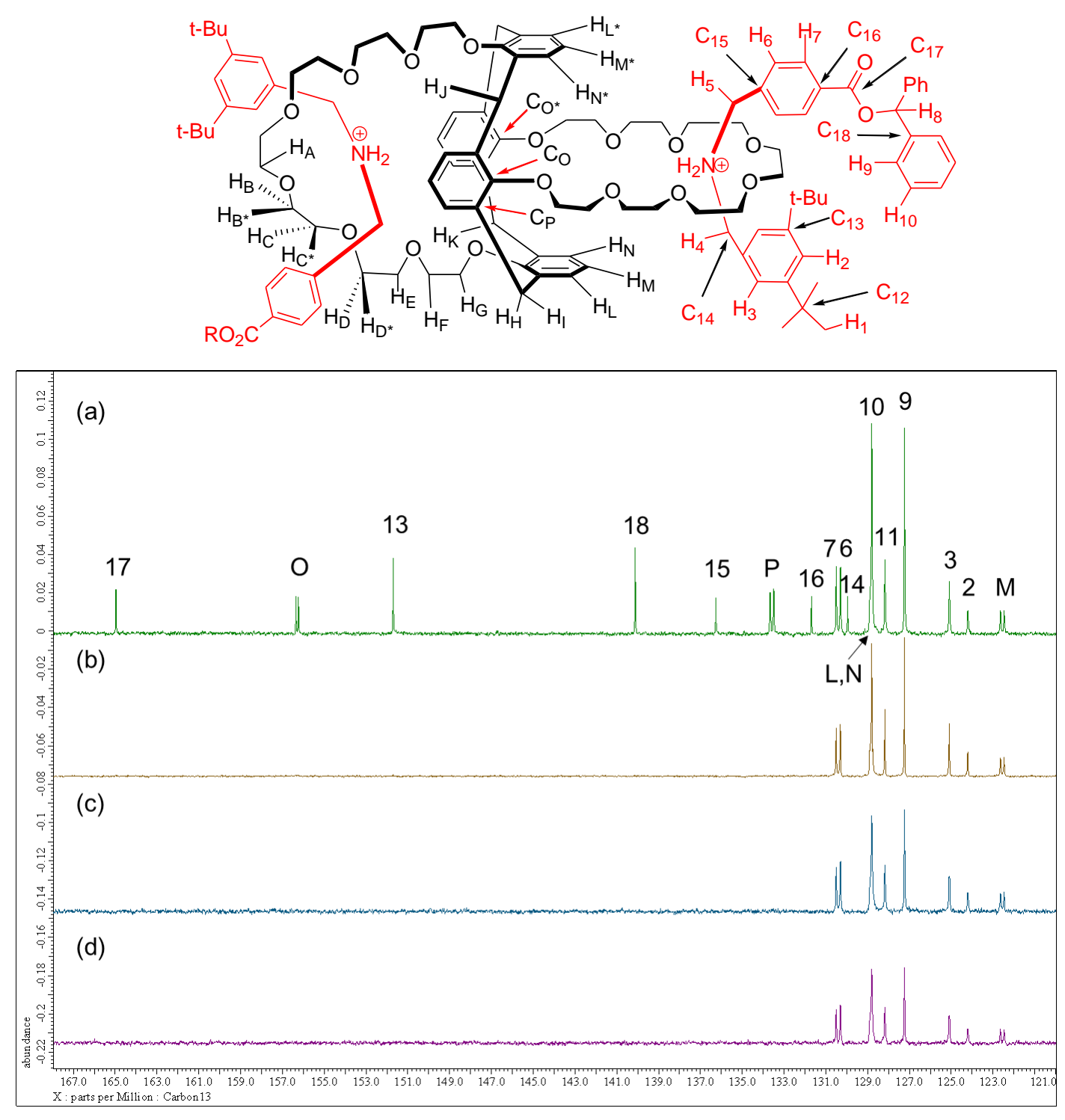


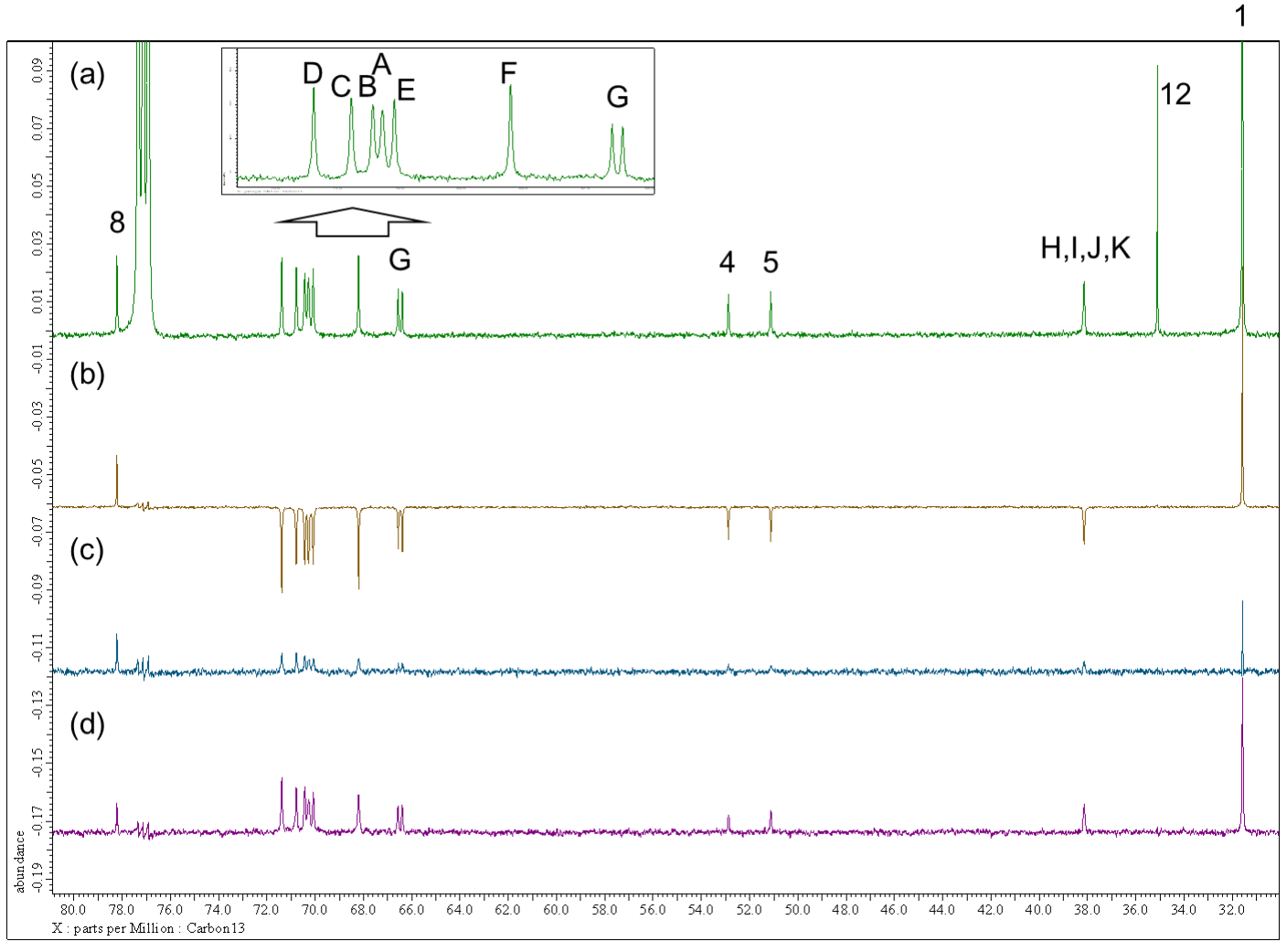

Figure S2a. ${ }^{13} \mathrm{C}$ NMR and DEPT spectra $\left(150 \mathrm{MHz}, \mathrm{CDCl}_{3}, 21{ }^{\circ} \mathrm{C}\right)$ of the [3]rotaxane 1: (a) ${ }^{13} \mathrm{C}$ NMR, (b) 135, (c) 90, and (d) $45^{\circ}$ DEPT spectra. 

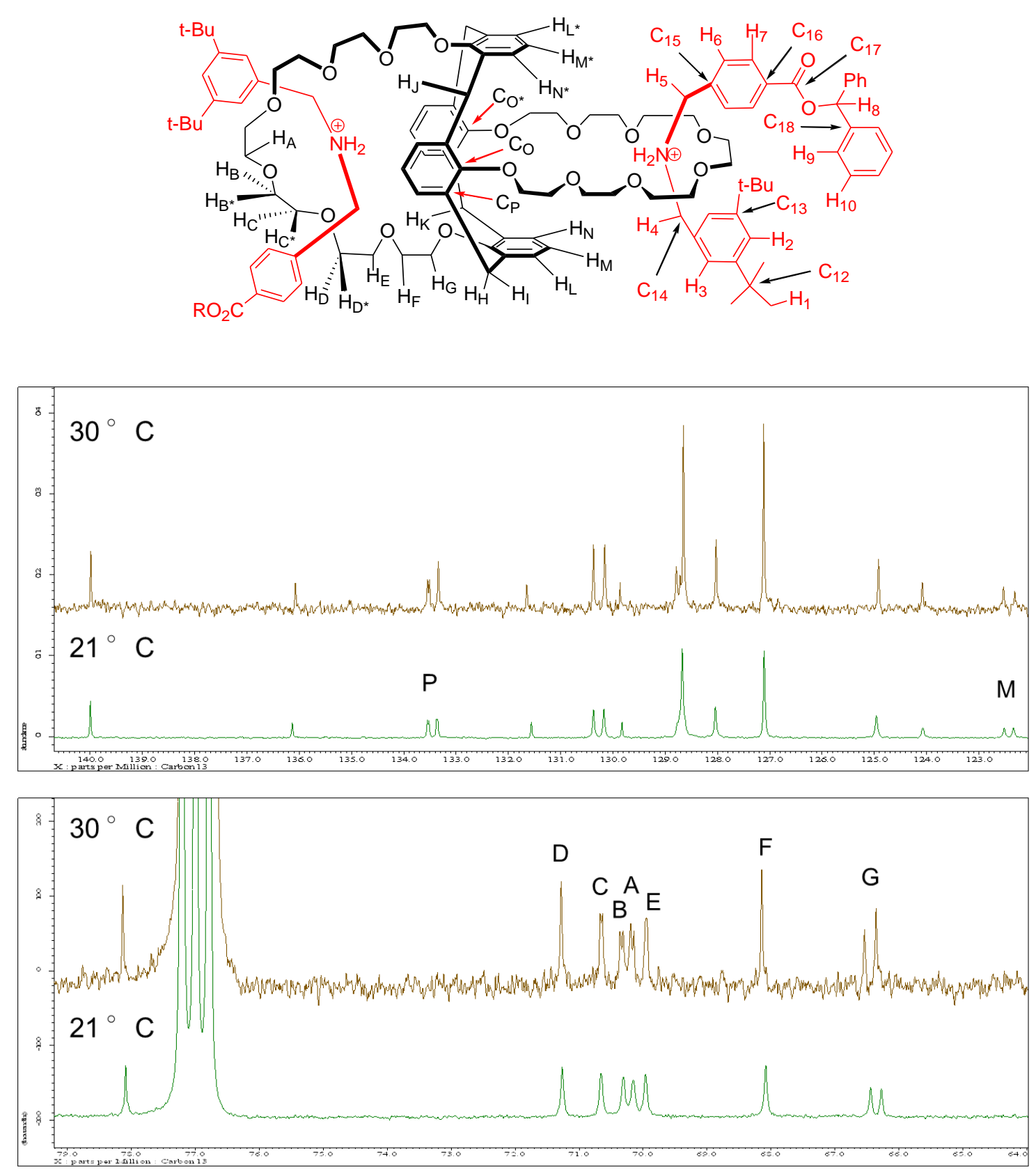

Figure S2b. ${ }^{13} \mathrm{C}$ NMR spectra $\left(150 \mathrm{MHz}, \mathrm{CDCl}_{3}\right)$ of the [3]rotaxane 1 at $21{ }^{\circ} \mathrm{C}$ (lower) and at $30{ }^{\circ} \mathrm{C}$ (upper). 

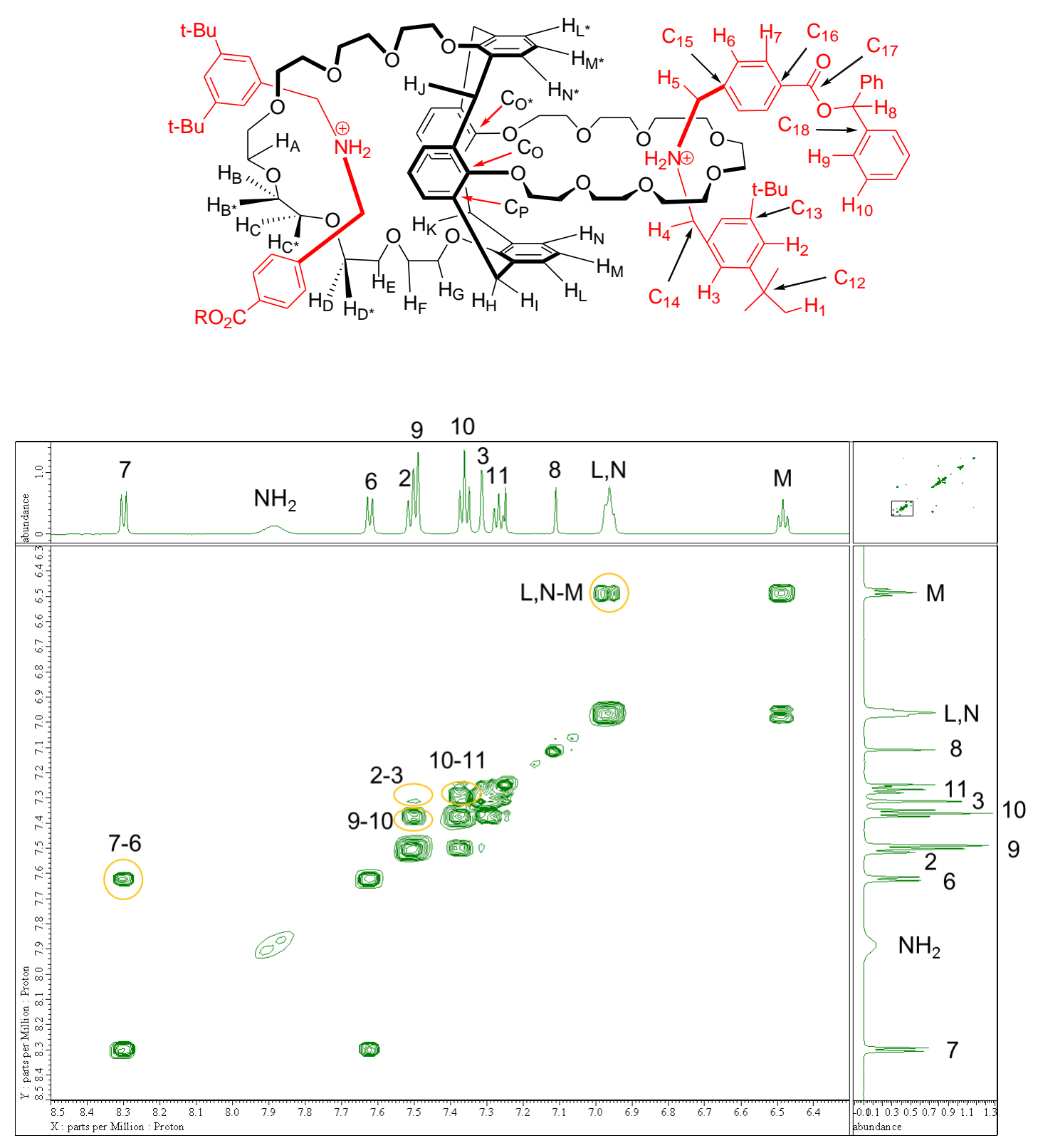


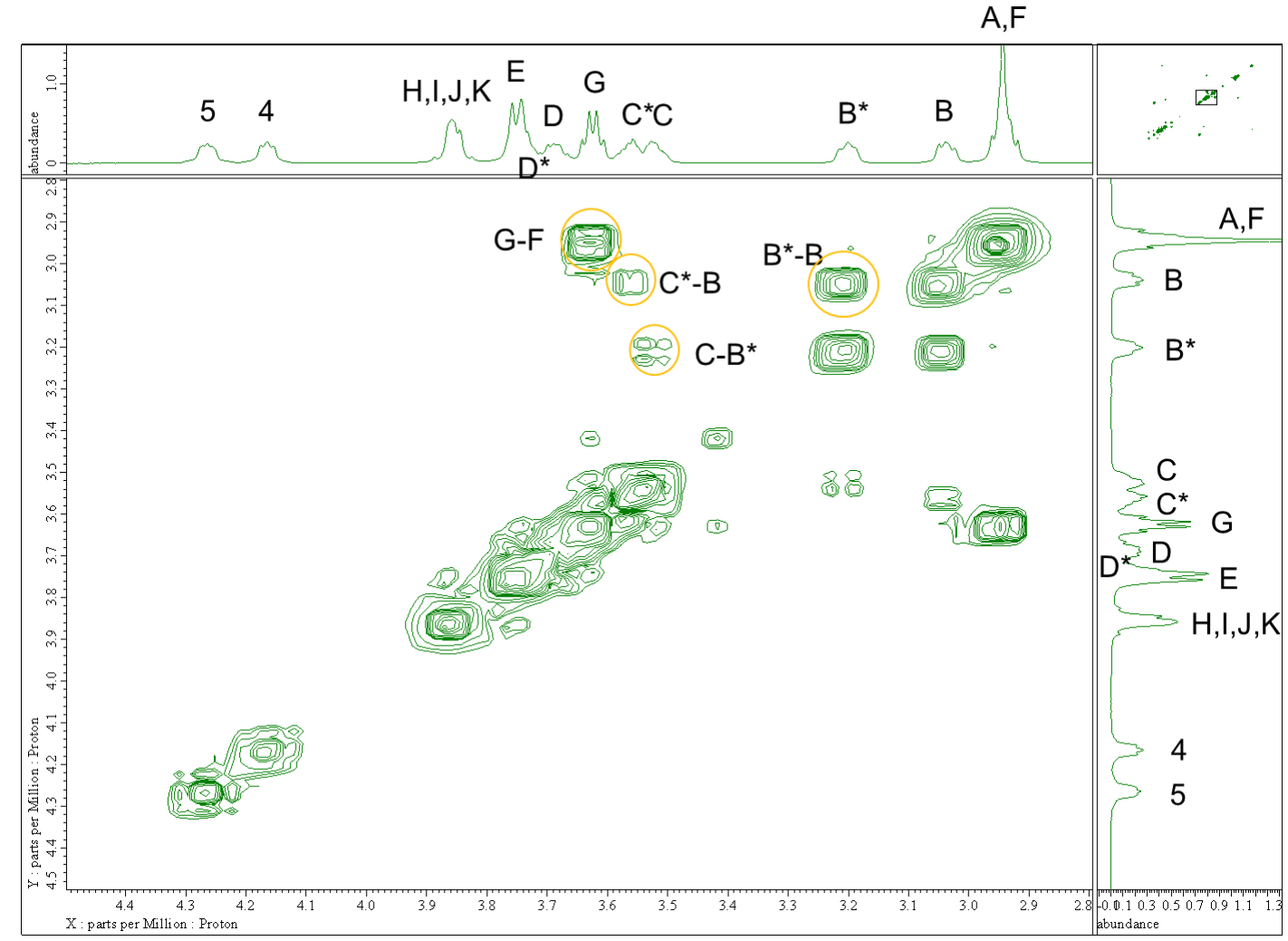

Figure S3. COSY spectrum $\left(600 \mathrm{MHz}, \mathrm{CDCl}_{3}, 25^{\circ} \mathrm{C}\right)$ of the [3]rotaxane 1. 

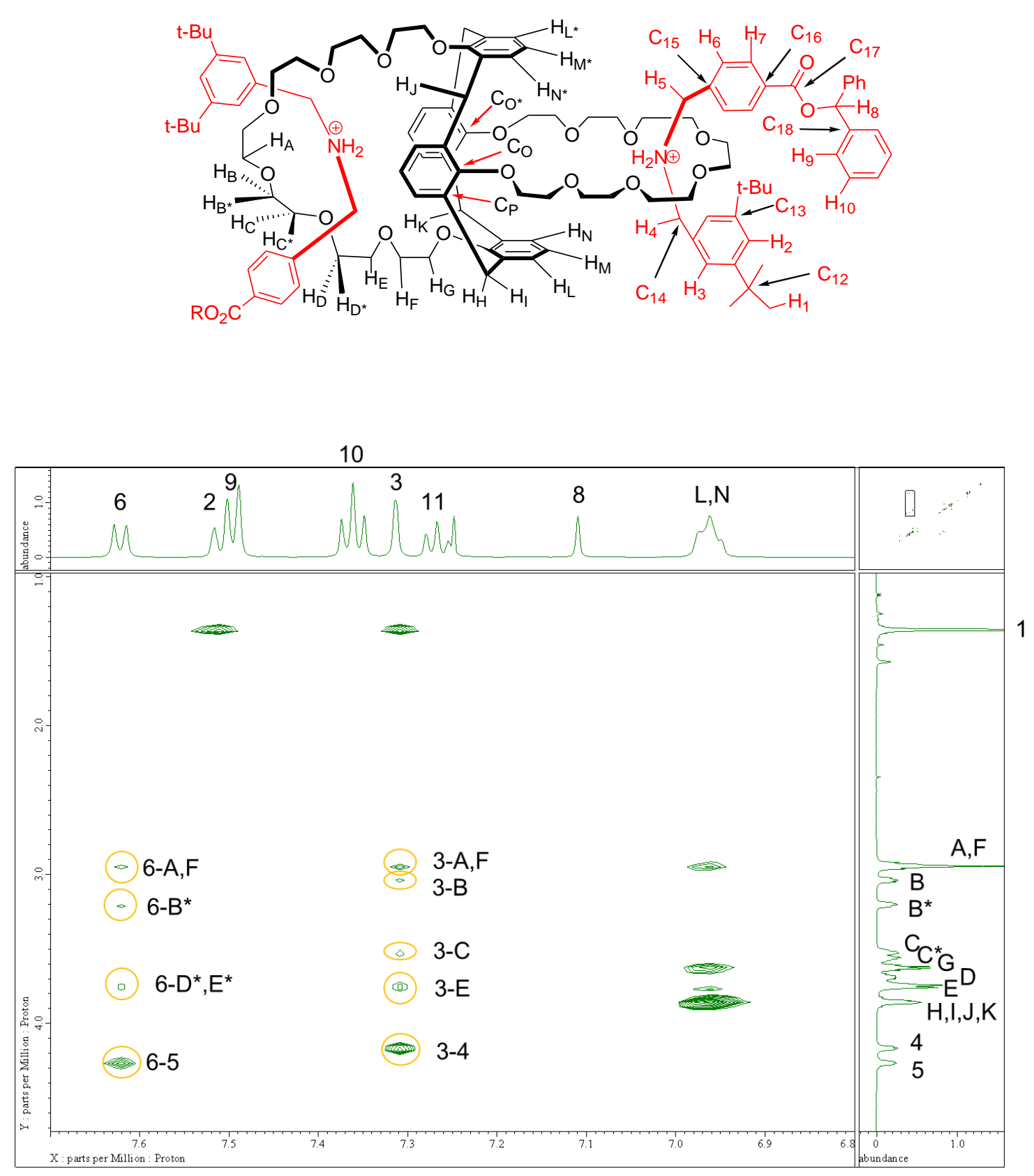


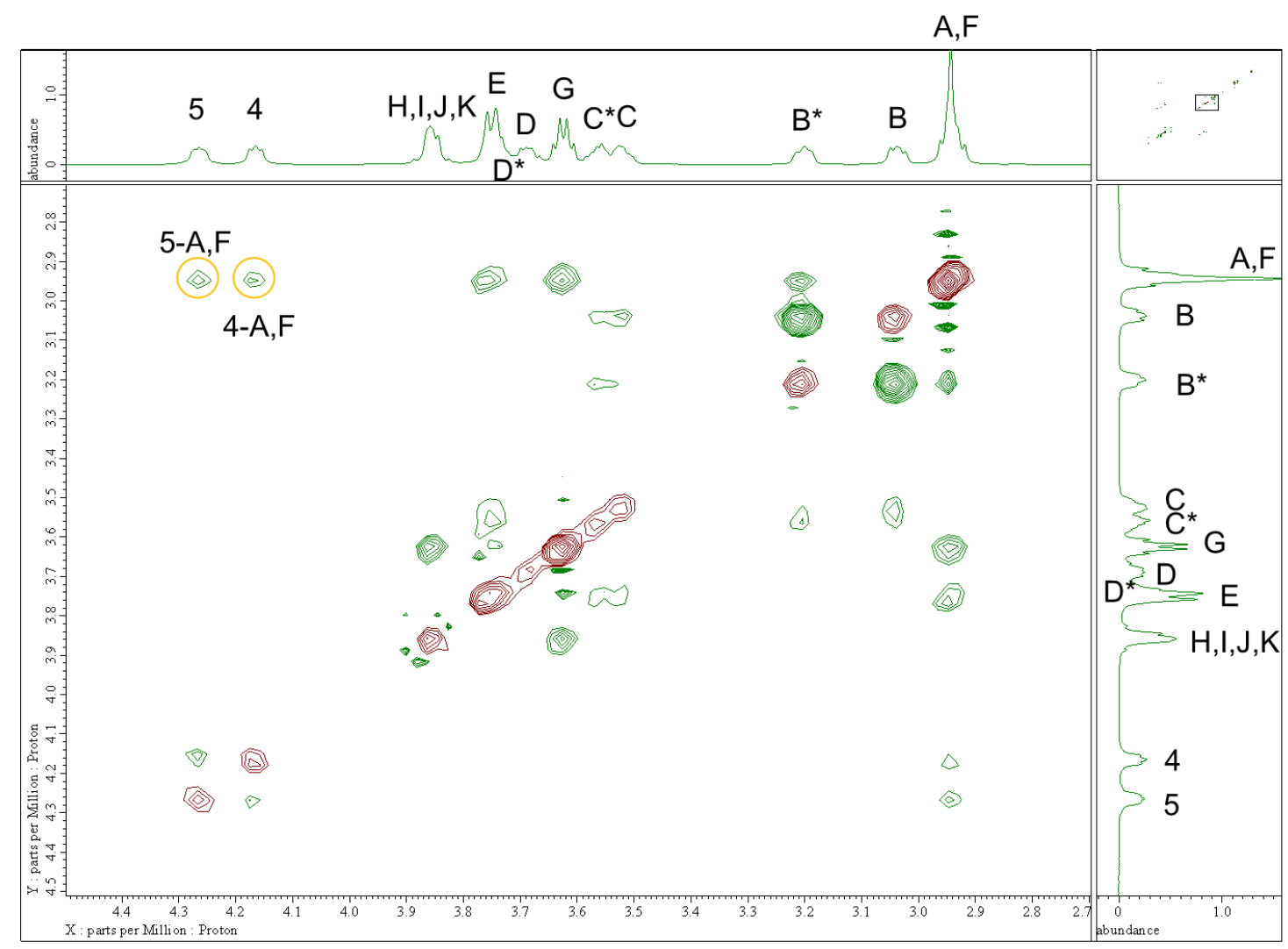

Figure S4. ROESY spectrum $\left(600 \mathrm{MHz}, \mathrm{CDCl}_{3}, 25^{\circ} \mathrm{C}\right)$ of the [3]rotaxane 1. 

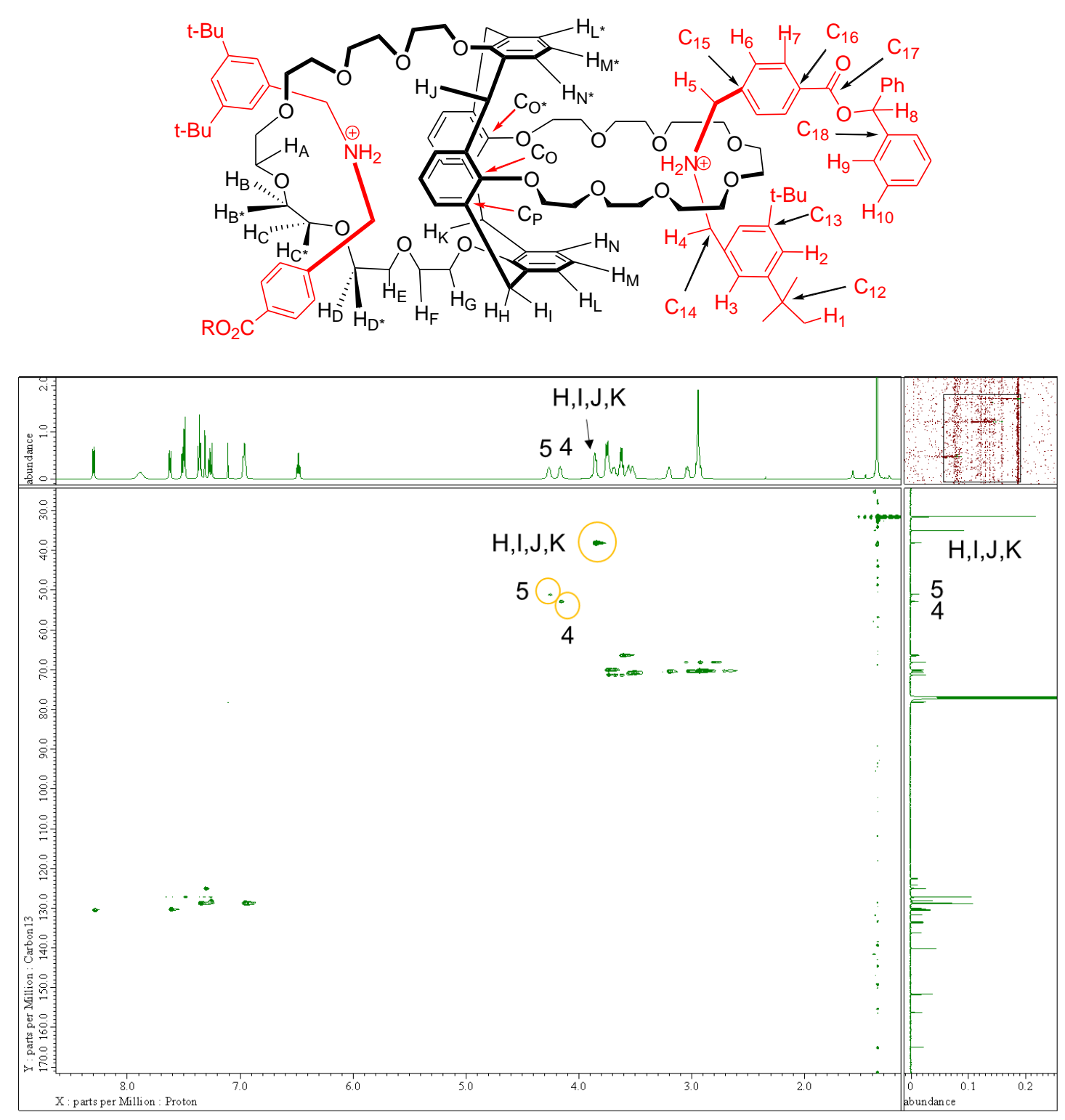

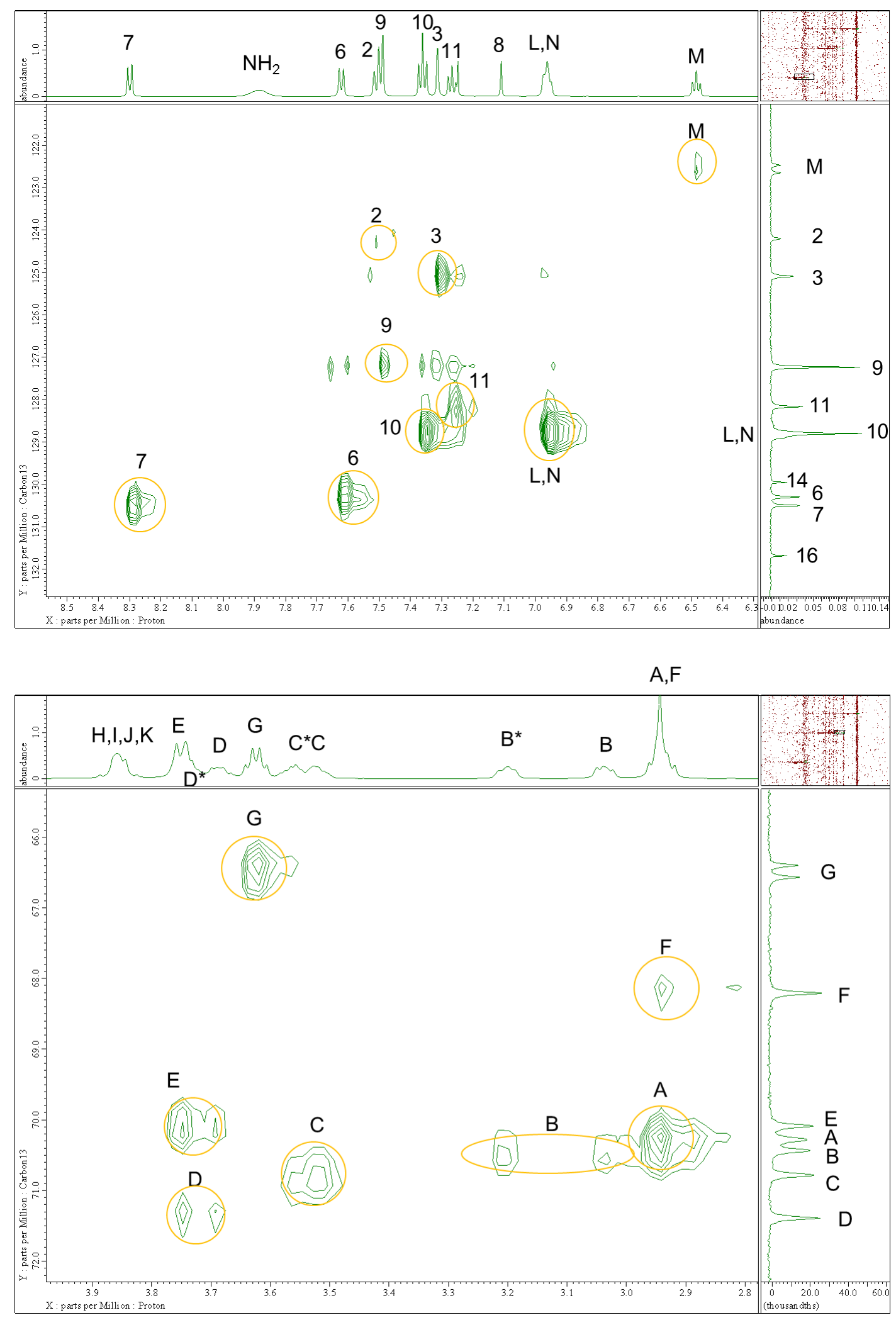

Figure S5. HSQC spectrum $\left(600 \mathrm{MHz}, \mathrm{CDCl}_{3}, 25^{\circ} \mathrm{C}\right)$ of the [3] rotaxane 1. 

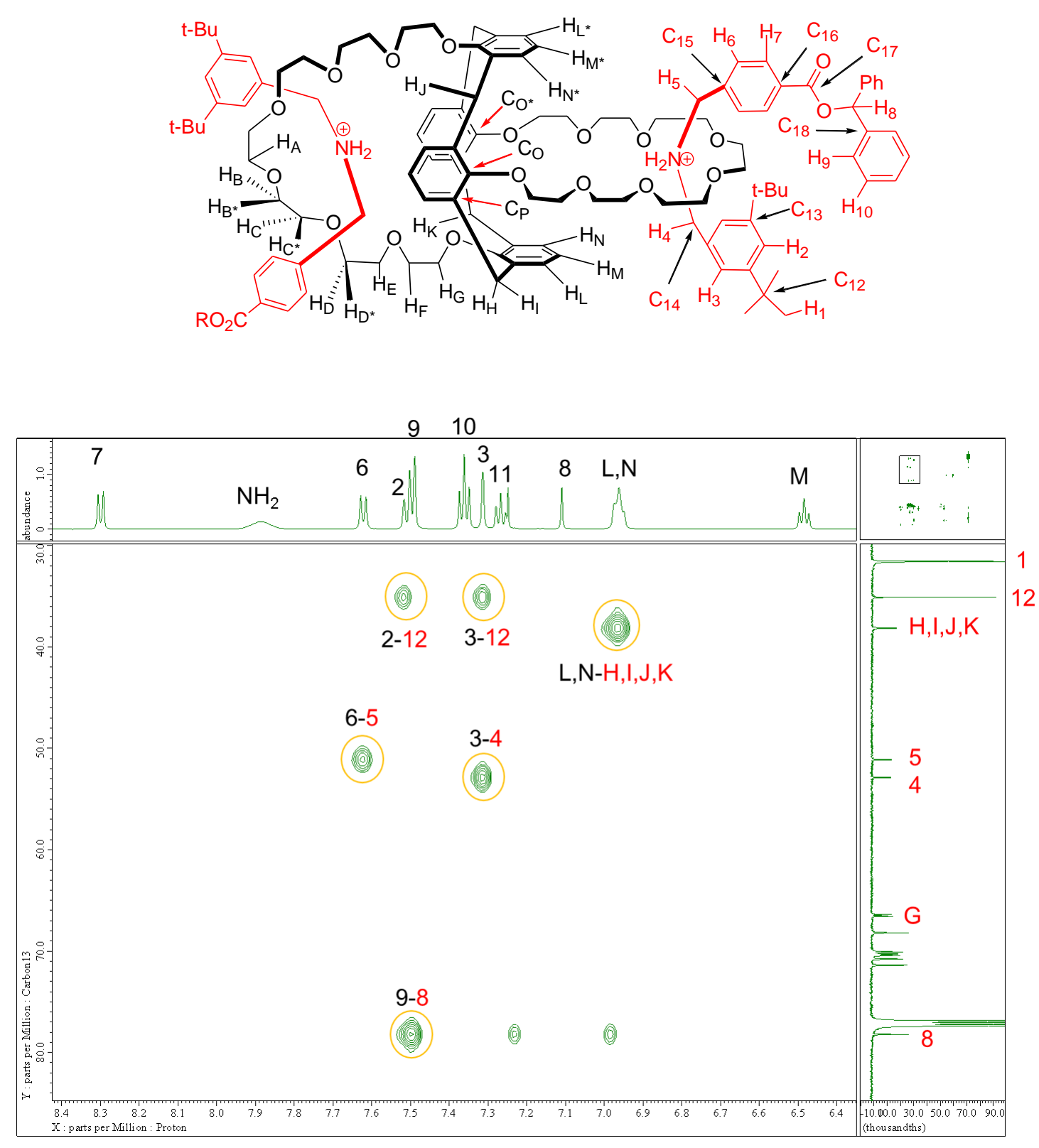

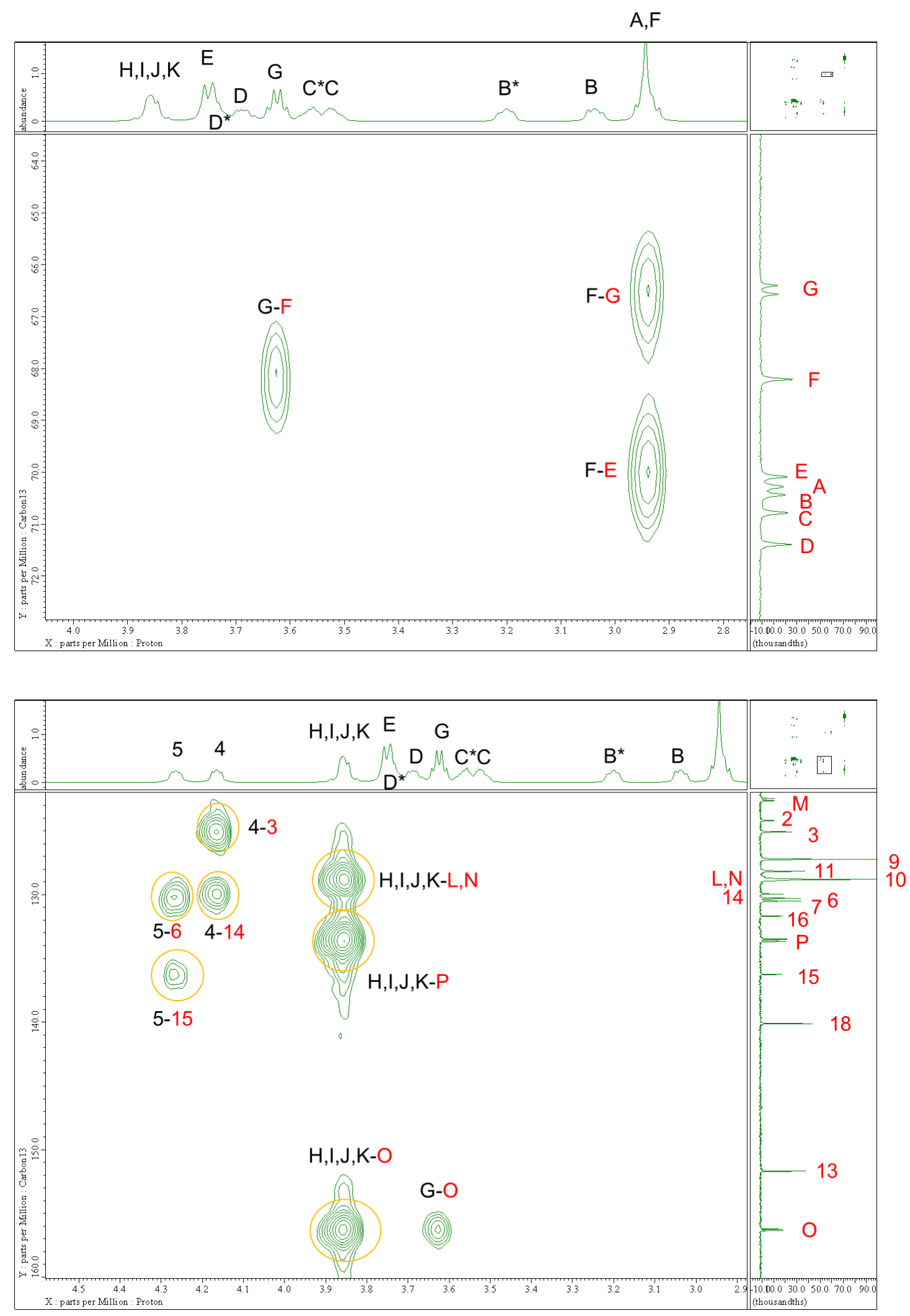


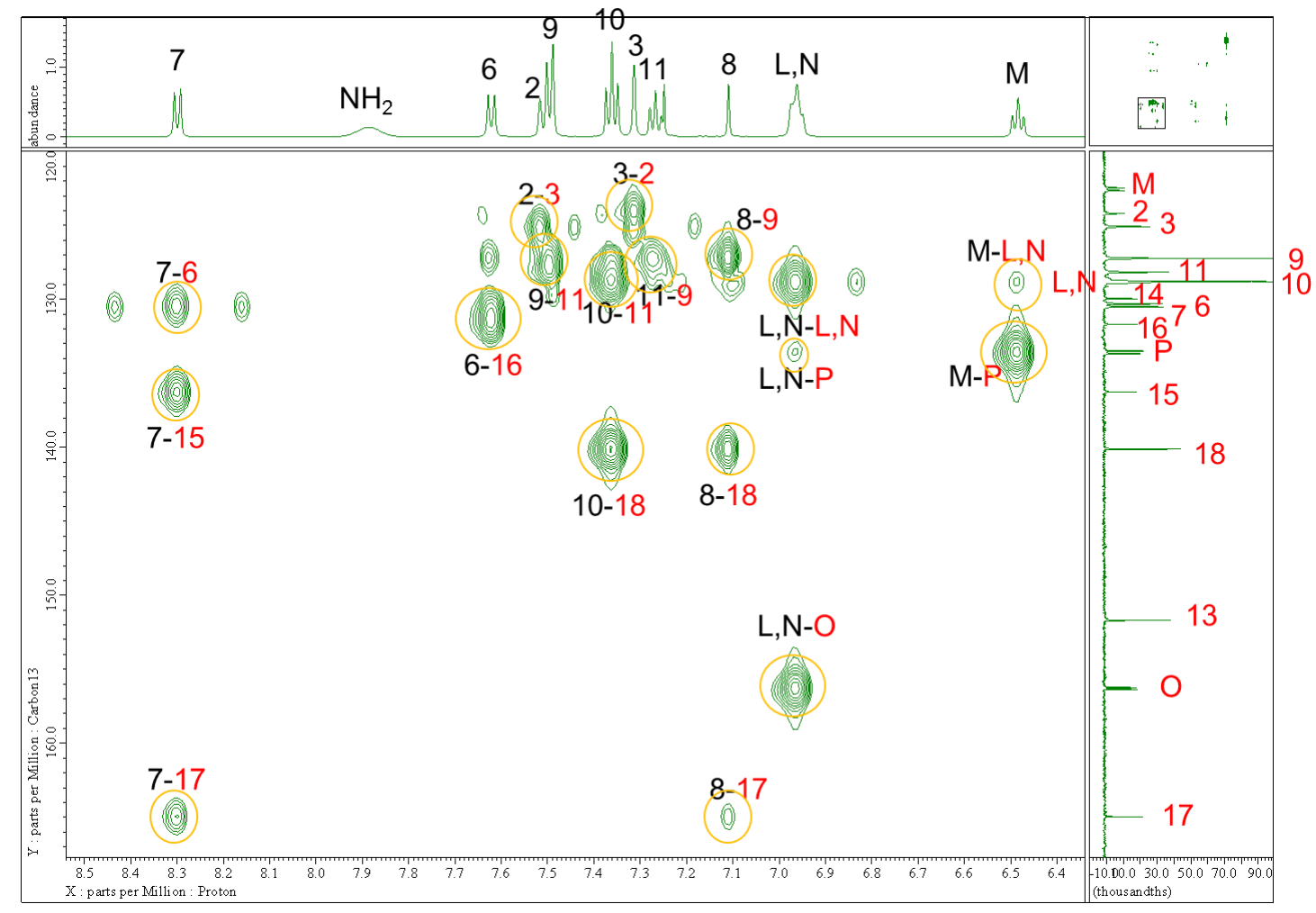

Figure S6. $\mathrm{HMBC}$ spectrum $\left(600 \mathrm{MHz}, \mathrm{CDCl}_{3}\right)$ of the [3] rotaxane $\mathbf{1}$. 


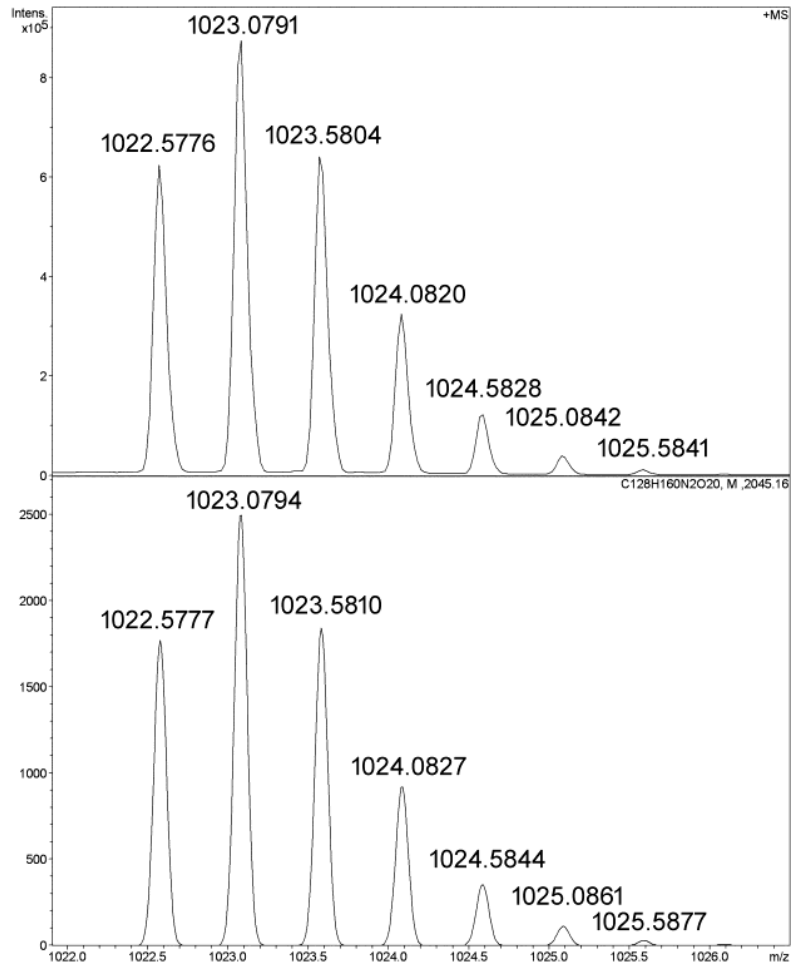

Figure S7. Mass spectrum of the [3]rotaxane 1, experimental (top) and calculated (bottom) isotopic patterns. 
Table S1. Crystal data and structure refinement parameters for the [3] rotaxane $\mathbf{1 .}$

Empirical Formula

Formula Weight

Temperature

Crystal System

Lattice Type

Space Group

Lattice Parameters

Volume

$Z$ value

$\rho_{\text {calc }}$

$F(000)$

Crystal Color

Radiation

$2 \theta_{\max }$

Number of Reflections Measured

Goodness-of-Fit on $F^{4}$

Residuals: $R_{1}[I>2.00 \sigma(I)]$

Residuals: $\mathrm{w} R_{2}$ (All reflections)
$\mathrm{C}_{128} \mathrm{H}_{160} \mathrm{~F}_{12} \mathrm{~N}_{2} \mathrm{O}_{20} \mathrm{P}_{2}$

2336.60

$-100.0^{\circ} \mathrm{C}$

monoclinic

Primitive

$\mathrm{P} 21 / \mathrm{c}$ (\#14)

$a=14.83241 \AA$

$b=55.18274 \AA$

$c=16.74578 \AA$

$\beta=97.89100^{\circ}$

$13576.51960 \AA^{3}$

4

$1.143 \mathrm{~g} / \mathrm{cm}^{3}$

4960.00

colorless

Synchrotron $(\lambda=0.81042 \AA)$

$58.4^{\circ}$

Total: 137626

Unique: $24830\left(R_{\text {int }}=0.1132\right)$

1.346

0.1580

0.4730 


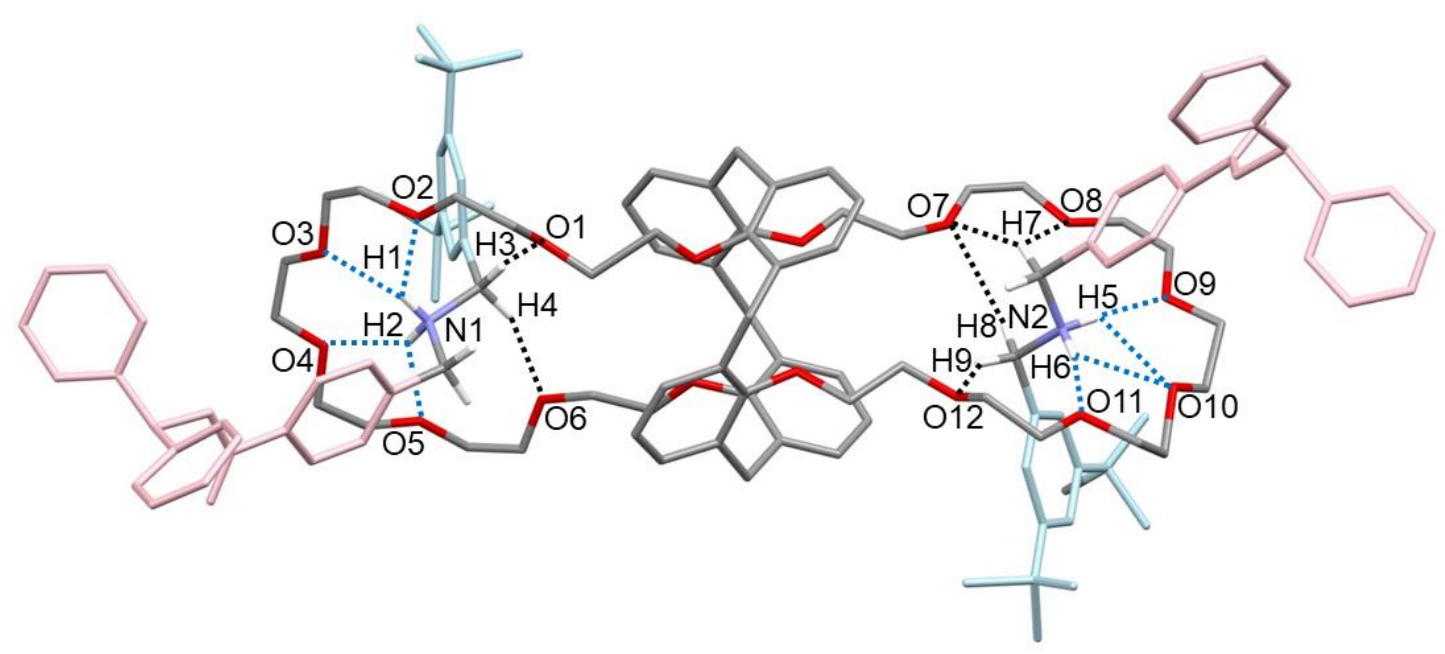

Figure S8a. Crystal structure of the racemic [3] rotaxane 1. Hydrogen bonding distances of $\mathrm{NH} \bullet \bullet \mathrm{O}$ (blue dotted lines) are $2.53 \AA[\mathrm{H}(1) \bullet \bullet \mathrm{O}(2)], 2.01 \AA[\mathrm{H}(1) \bullet \bullet \mathrm{O}(3)], 2.15 \AA$ $[\mathrm{H}(2) \bullet \bullet \mathrm{O}(4)], 2.37 \AA[\mathrm{H}(2) \bullet \bullet \mathrm{O}(5)], 2.00 \AA[\mathrm{H}(5) \bullet \bullet \mathrm{O}(9)], 2.59 \AA[\mathrm{H}(5) \bullet \bullet \mathrm{O}(10)], 2.30$ $\AA[\mathrm{H}(6) \cdots \bullet \mathrm{O}(10)], 2.08 \AA[\mathrm{H}(6) \cdots \cdot \mathrm{O}(11)]$, and $\mathrm{CH} \bullet \bullet \mathrm{O}$ (black dotted lines) are $2.33 \AA$ $[\mathrm{H}(3) \bullet \bullet \mathrm{O}(1)], 2.17 \AA[\mathrm{H}(4) \bullet \bullet \mathrm{O}(6)], 2.45 \AA[\mathrm{H}(7) \cdots \bullet \mathrm{O}(7)], 2.70 \AA[\mathrm{H}(7) \cdots \bullet \mathrm{O}(8)], 2.64 \AA$ $[\mathrm{H}(8) \bullet \bullet \mathrm{O}(7)], 2.45 \AA[\mathrm{H}(9) \cdots \bullet \mathrm{O}(12)]$. Hydrogen atoms and $\mathrm{PF}_{6}{ }^{-}$ions are omitted for clarity. 


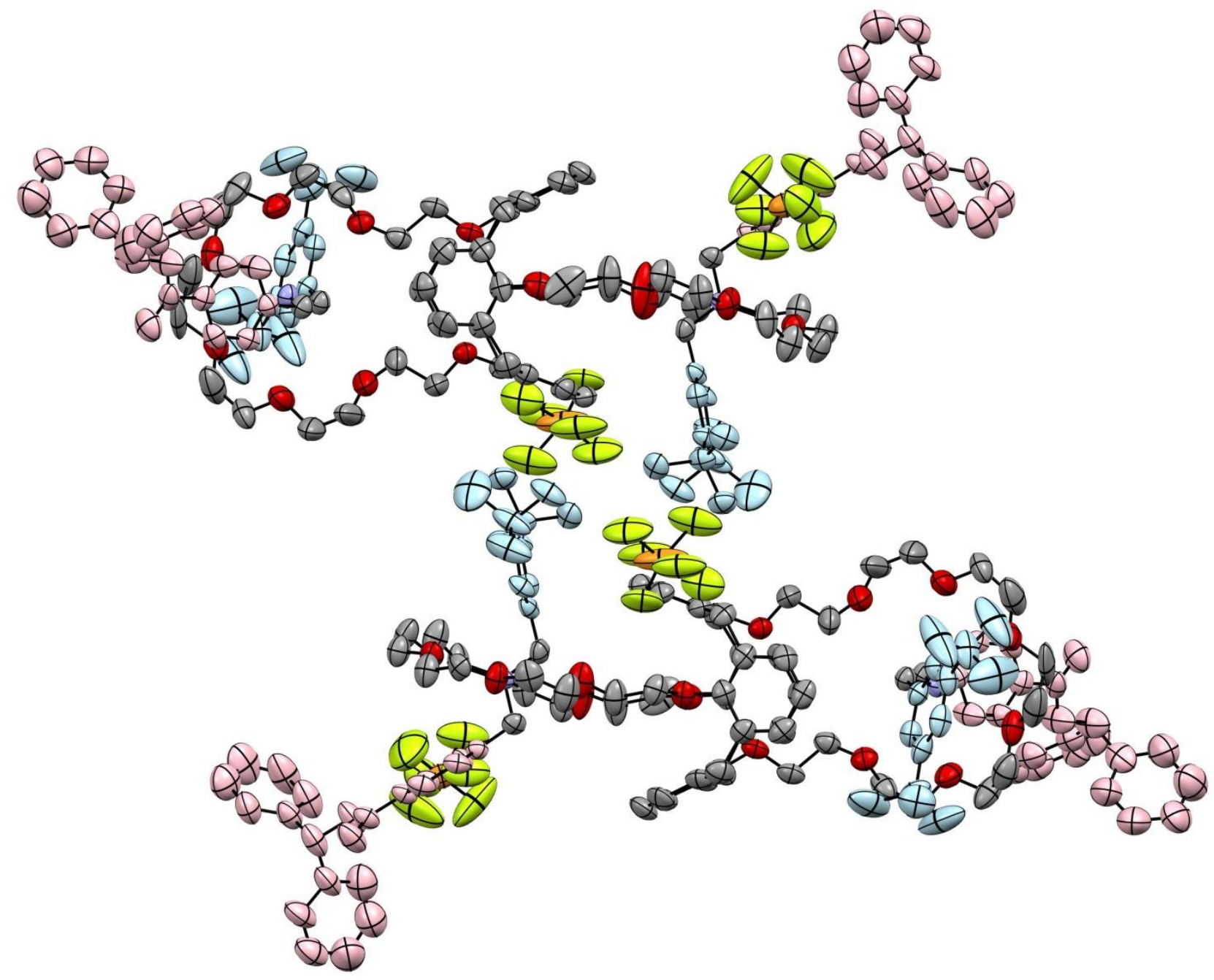

Figure S8b. Packing structure of the racemic [3] rotaxane 1. Hydrogen atoms have been omitted for clarity. Thermal ellipsoids are displayed at the $30 \%$ probability level. Gray: carbon; blue: nitrogen; red: oxygen; green: fluorine; orange: phosphorus. The axle components are colored in pale blue and pale red. The crystal was obtained via slow evaporation of $\mathrm{CHCl}_{3} / \mathrm{AcOEt}=1: 1[\mathrm{v} / \mathrm{v}]$ at room temperature. 
(a)
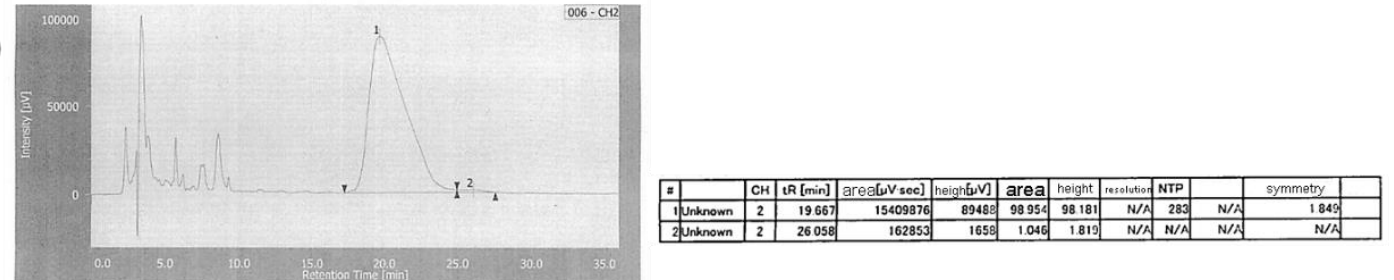

(b)
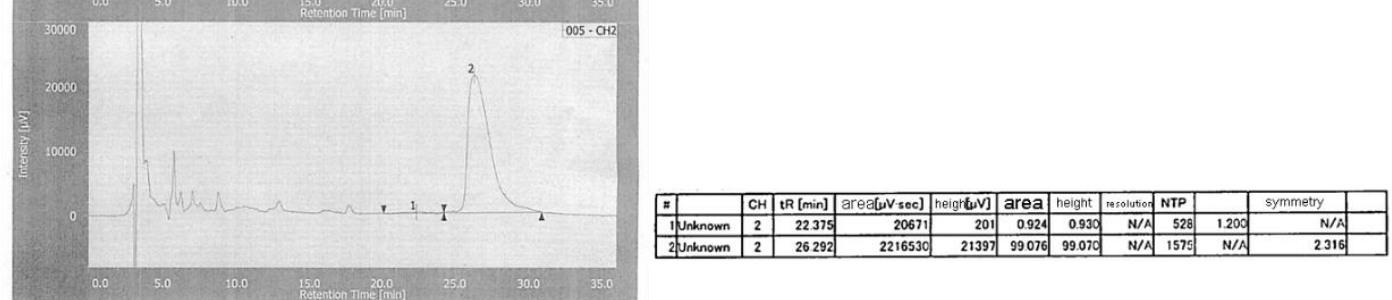

(c)
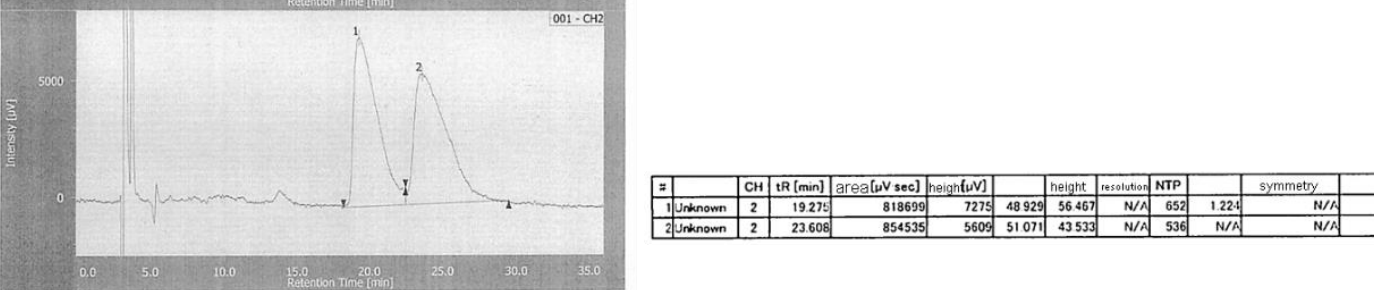

Figure S9. Chiral HPLC analysis of the [3] rotaxane 1. (a, b) The two enantiomers of 1 after separation; (c) racemic mixture.

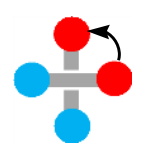

(S)-isomer

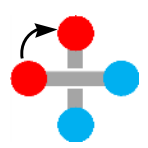

$(R)$-isomer

Figure S10. Cartoon illustration of description of the absolute configurations of the [3] rotaxanes 1. The rule of axial chirality is adopted for description of the absolute configuration of each enantiomer on an assignment for the relative direction of the axle components in the [3] rotaxane. 


\section{Computational Details}

All calculations were performed using Gaussian 16 package at the M06-2X/6-31G(d) level. ${ }^{\mathrm{S} 4}$ Geometry optimization of a model [3] rotaxane with the $(R)$-configuration $((R)$ 1') was carried out using the X-ray geometry of $\mathbf{1}$ as an initial structure. tert-Butyl groups and diphenylmethyl groups in $\mathbf{1}$ were replaced with methyl groups and counter anions were omitted to reduce computational costs. Vibrational frequency computations of the optimized structure verified the nature of the stationary points. Excitation wavelengths, oscillator strengths, and rotational strengths were calculated using the TD-DFT approach. The spectra were simulated by superimposing Gaussian bands with a half-width at half height of $2800 \mathrm{~cm}^{-1}$.

Table S2. Selected TD-DFT results for $(R)-1$ '.

\begin{tabular}{lcccl}
\hline & $\lambda / \mathrm{nm}$ & $f$ & $R^{[\mathrm{a}]}$ & Major composition (weight, \% $)^{[\mathrm{b}]}$ \\
\hline Excited state 11 & 212 & 0.56 & 727.63 & $408 \rightarrow 423(60.0 \%)$ \\
Excited state 12 & 212 & 0.36 & -812.05 & $410 \rightarrow 424(30.8 \%), 409 \rightarrow 424(21.1 \%)$, \\
& & & & $411 \rightarrow 424(20.2 \%), 408 \rightarrow 423(13.6 \%)$ \\
\hline
\end{tabular}

[a] The unit of $R$ is $10^{-40} \mathrm{erg}$ esu cm Gauss ${ }^{-1}$. [b] Compositions with more than $10 \%$ were shown.

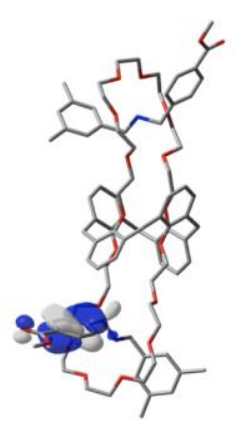

408

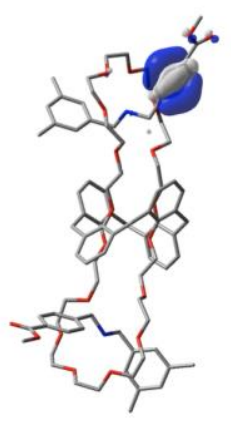

409

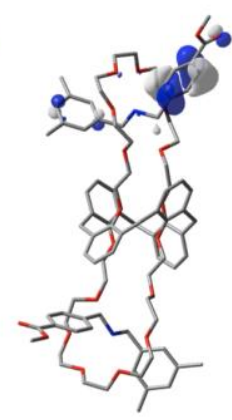

410

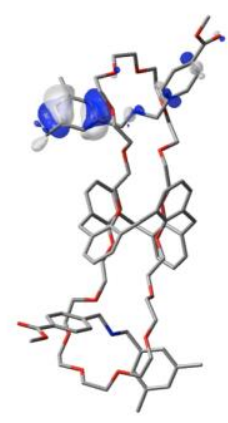

411

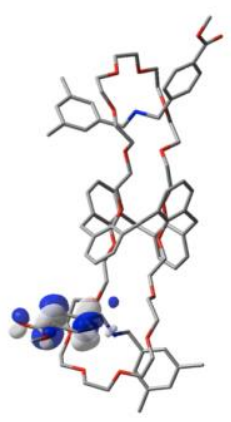

423

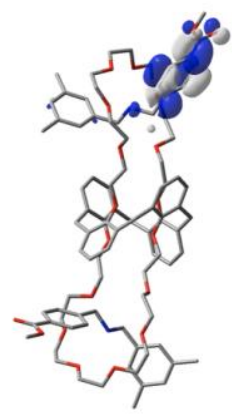

424

Figure S11. Selected molecular orbitals for $(R)-1$ ' (isovalue $=0.04)$. 


\section{Cartesian Coordinates (in Å) and Energies}

\begin{tabular}{|c|c|c|c|c|c|c|c|}
\hline \multirow{2}{*}{\multicolumn{4}{|c|}{$(R)-1 '$}} & \multirow{2}{*}{$\begin{array}{l}\mathrm{C} \\
\mathrm{C}\end{array}$} & \multirow{2}{*}{$\begin{array}{l}1.375727 \\
2.661727\end{array}$} & \multirow{2}{*}{$\begin{array}{l}2.031316 \\
2.488299\end{array}$} & \multirow{2}{*}{$\begin{array}{l}-0.011269 \\
-0.322479\end{array}$} \\
\hline & & & & & & & \\
\hline $\mathrm{O}$ & -1.704814 & -2.509558 & 0.948479 & $\mathrm{C}$ & 3.22777 & 2.257358 & -1.571949 \\
\hline $\mathrm{O}$ & -5.04987 & -2.298897 & 2.07933 & $\mathrm{C}$ & 2.501894 & 1.560603 & -2.535694 \\
\hline $\mathrm{O}$ & -7.841139 & -2.18408 & 2.317499 & 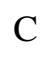 & 0.746084 & 2.453861 & 1.308606 \\
\hline $\mathrm{O}$ & -9.478585 & 0.149374 & 1.603508 & $\mathrm{C}$ & -0.276066 & 1.557053 & 1.974878 \\
\hline $\mathrm{O}$ & -8.850209 & 2.285417 & -0.278825 & $\mathrm{C}$ & 0.068849 & 0.257718 & 2.356173 \\
\hline $\mathrm{O}$ & -6.388932 & 2.534709 & -1.388747 & $\mathrm{C}$ & -0.820656 & -0.588547 & 3.020306 \\
\hline $\mathrm{O}$ & -4.088048 & 1.188841 & -0.857987 & $F$ & -2.102685 & -0.106811 & 3.297135 \\
\hline $\mathrm{O}$ & -0.523064 & 0.745235 & -0.664018 & $\mathrm{C}$ & -2.467273 & 1.189769 & 2.945656 \\
\hline $\mathrm{O}$ & 1.330774 & -0.189852 & 2.033856 & $\mathrm{C}$ & -1.555204 & 2.016132 & 2.28991 \\
\hline $\mathrm{O}$ & 4.771304 & -0.031463 & 3.117435 & 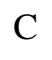 & -0.375398 & -1.964687 & 3.469359 \\
\hline $\mathrm{O}$ & 7.210962 & 1.454996 & 2.960478 & $\mathrm{C}$ & -2.784675 & -3.125379 & 1.633111 \\
\hline $\mathrm{O}$ & 9.496925 & 0.876018 & 1.254272 & $\mathrm{C}$ & -4.043965 & -2.447575 & 1.102996 \\
\hline $\mathrm{O}$ & 9.517006 & -0.537577 & -1.296042 & 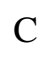 & -5.823239 & -3.449746 & 2.336257 \\
\hline $\mathrm{O}$ & 7.266869 & -1.577767 & -2.34398 & $\mathrm{C}$ & -7.032202 & -3.013656 & 3.132058 \\
\hline $\mathrm{O}$ & 4.543975 & -1.895355 & -1.55682 & $\mathrm{C}$ & -9.007857 & -1.759262 & 2.992991 \\
\hline $\mathrm{O}$ & 0.93209 & -1.66442 & -1.391778 & 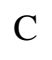 & -9.968016 & -1.116025 & 2.021695 \\
\hline $\mathrm{C}$ & -0.465221 & -3.069607 & 1.180624 & 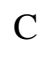 & -10.412257 & 0.822171 & 0.765974 \\
\hline $\mathrm{C}$ & 0.091852 & -3.835578 & 0.153649 & $\mathrm{C}$ & -9.971084 & 2.254387 & 0.58036 \\
\hline $\mathrm{C}$ & 1.34526 & -4.41034 & 0.355808 & $\mathrm{H}$ & -9.71771 & 2.689642 & 1.559211 \\
\hline $\mathrm{C}$ & 2.034456 & -4.210048 & 1.549315 & $\mathrm{H}$ & -10.795677 & 2.836866 & 0.144569 \\
\hline $\mathrm{C}$ & 1.46988 & -3.422695 & 2.54678 & $\mathrm{C}$ & -8.27069 & 3.572753 & -0.360394 \\
\hline $\mathrm{C}$ & 0.212047 & -2.838311 & 2.38067 & 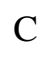 & -7.321715 & 3.590993 & -1.536622 \\
\hline $\mathrm{C}$ & -0.6826 & -4.06679 & -1.122065 & $\mathrm{C}$ & -5.318563 & 2.599478 & -2.310355 \\
\hline $\mathrm{C}$ & -1.055417 & -2.862578 & -1.970646 & $\mathrm{C}$ & -4.512118 & 1.324853 & -2.200058 \\
\hline $\mathrm{C}$ & -0.202876 & -1.769532 & -2.159589 & $\mathrm{C}$ & -2.833592 & 0.547362 & -0.682863 \\
\hline $\mathrm{C}$ & -0.507063 & -0.745077 & -3.068667 & J & -1.675231 & 1.465014 & -1.040871 \\
\hline $\mathrm{C}$ & -1.710524 & -0.818412 & -3.77024 & $\mathrm{C}$ & 2.309433 & -0.020154 & 3.04346 \\
\hline $\mathrm{C}$ & -2.592705 & -1.878216 & -3.574208 & $\mathrm{C}$ & 3.629025 & -0.131809 & 2.299754 \\
\hline $\mathrm{C}$ & -2.26045 & -2.88831 & -2.680027 & 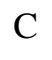 & 4.956383 & 1.231217 & 3.719049 \\
\hline $\mathrm{C}$ & 0.444113 & 0.397238 & -3.373729 & $\mathrm{C}$ & 6.406572 & 1.358035 & 4.121736 \\
\hline $\mathrm{C}$ & 1.224829 & 1.071739 & -2.260737 & $\mathrm{C}$ & 8.558134 & 1.731836 & 3.287556 \\
\hline $\mathrm{C}$ & 0.687512 & 1.301201 & -0.985707 & $\mathrm{C}$ & 9.339911 & 2.049129 & 2.037355 \\
\hline
\end{tabular}




\begin{tabular}{|c|c|c|c|c|c|c|c|}
\hline $\mathrm{C}$ & 10.426082 & 1.072766 & 0.196414 & $\mathrm{C}$ & -10.068576 & -1.639286 & -2.758374 \\
\hline $\mathrm{C}$ & 10.655824 & .236425 & -0.516475 & $\mathrm{C}$ & -9.699219 & -2.556174 & -1.774741 \\
\hline $\mathrm{C}$ & 9.601854 & -1.817693 & -1.892359 & $\mathrm{C}$ & -8.585408 & -2.307918 & -0.980042 \\
\hline 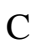 & 8.511438 & -1.919006 & -2.93284 & $\mathrm{C}$ & -11.279817 & -1.955944 & -3.578524 \\
\hline $\mathrm{C}$ & 6.196316 & -1.718349 & -3.258586 & $\mathrm{C}$ & -12.671507 & -1.28005 & -5.325961 \\
\hline $\mathrm{C}$ & 4.934129 & -1.130093 & -2.677427 & $\mathrm{H}$ & -12.545617 & -2.221646 & -5.863288 \\
\hline $\mathrm{C}$ & 3.244475 & -1.593773 & -1.066974 & $\mathrm{C}$ & -6.645574 & -0.882372 & -0.271456 \\
\hline $\mathrm{C}$ & 2.13877 & -2.06978 & -2.00233 & $\mathrm{C}$ & -5.935678 & 0.627748 & 1.635268 \\
\hline $\mathrm{O}$ & 7.945483 & 6.638381 & -1.807351 & $\mathrm{C}$ & -6.110637 & 2.048191 & 2.10247 \\
\hline $\mathrm{O}$ & 8.700961 & 5.359821 & -3.494656 & $\mathrm{C}$ & -7.169616 & 2.386816 & 2.945351 \\
\hline $\mathrm{N}$ & 7.098742 & -0.35268 & 0.24894 & $\mathrm{C}$ & $-7.3^{\circ}$ & 3.711489 & 3.340425 \\
\hline $\mathrm{C}$ & 7.599019 & 4.280519 & -1.713405 & $\mathrm{C}$ & -6.489047 & 4.687539 & 2.869363 \\
\hline$C$ & 6.945895 & 4.363198 & -0.48369 & $\mathrm{C}$ & -5.425102 & 4.373366 & 2.019851 \\
\hline $\mathrm{C}$ & 6.478806 & 3.206816 & 0.129768 & $\mathrm{C}$ & 29 & 22 & 1.633362 \\
\hline $\mathrm{C}$ & 6.644866 & 1.963195 & -0.485843 & $\mathrm{C}$ & -8.500327 & 4.081143 & 4.26897 \\
\hline $\mathrm{C}$ & 7.301588 & 1.886123 & -1.718996 & $\mathrm{C}$ & -4.477893 & 5.445692 & 1.543969 \\
\hline $\mathrm{C}$ & 7.781753 & 3.041233 & -2.32 & $\mathrm{H}$ & -12.730843 & -0.443407 & -6.019015 \\
\hline$c$ & 8.089041 & 5.556981 & -2.322566 & $\mathrm{H}$ & -13.569701 & -1.338383 & -4.708575 \\
\hline $\mathrm{C}$ & 9.191227 & 6.549203 & -4.125059 & $\mathrm{H}$ & -8.988025 & 5.00742 & 3.952582 \\
\hline $\mathrm{H}$ & 28 & 7.042448 & -3.479853 & $\mathrm{H}$ & -9.255564 & 3.291715 & 4.311447 \\
\hline$c$ & 6.086145 & 0.735066 & 0.18094 & $\mathrm{H}$ & -8.129507 & 4.239416 & 5.287079 \\
\hline $\mathrm{C}$ & 6.628153 & -1.551836 & 1.013884 & $\mathrm{H}$ & -5.012909 & 6.360343 & 1.273242 \\
\hline $\mathrm{C}$ & 7.629953 & -2.665067 & 0.887601 & $\mathrm{H}$ & -3.762212 & 5.709822 & 2.330118 \\
\hline $\mathrm{C}$ & 7.359485 & -3.751777 & 0.059056 & $\mathrm{H}$ & 8.366362 & 7.235926 & -4.323017 \\
\hline $\mathrm{C}$ & 8.290197 & -4.785206 & -0.085301 & $\mathrm{H}$ & 9.654166 & 6.221275 & -5.053414 \\
\hline $\mathrm{C}$ & 9.501245 & -4.689347 & 0.602323 & $\mathrm{H}$ & 11.953136 & -3.767009 & 1.497662 \\
\hline $\mathrm{C}$ & 9.80094 & -3.600647 & 1.428922 & $\mathrm{H}$ & 11.141024 & -4.265392 & 2.980214 \\
\hline $\mathrm{C}$ & 8.848927 & -2.590083 & 1.564895 & $\mathrm{H}$ & 11.282573 & -2.546705 & 2.595677 \\
\hline $\mathrm{C}$ & 7.986336 & -5.981328 & -0.950947 & $\mathrm{H}$ & 8.894225 & -6.385092 & -1.406335 \\
\hline$c$ & 11.115927 & -3.536959 & 2.163273 & $\mathrm{H}$ & 7.28397 & -5.725472 & -1.749112 \\
\hline $\mathrm{O}$ & -11.956365 & -2.941388 & -3.415161 & $\mathrm{H}$ & 7.533293 & -6.782978 & -0.357921 \\
\hline $\mathrm{O}$ & -11.525276 & -1.024274 & -4.506078 & $\mathrm{H}$ & -10.482917 & 0.312022 & -0.206267 \\
\hline $\mathrm{N}$ & -6.854649 & 0.39162 & 0.477153 & $\mathrm{H}$ & -11.401324 & 0.810226 & 1.243533 \\
\hline $\mathrm{C}$ & -7.830426 & -1.147542 & -1.165604 & $\mathrm{H}$ & -10.121847 & -1.769207 & 1.152393 \\
\hline $\mathrm{C}$ & -8.207064 & -0.232036 & -2.153796 & $\mathrm{H}$ & -10.934093 & -0.976418 & 2.526607 \\
\hline $\mathrm{C}$ & -9.322082 & -0.474747 & -2.948085 & $\mathrm{H}$ & -9.512645 & -2.622442 & 51321 \\
\hline
\end{tabular}




\begin{tabular}{|c|c|c|c|c|c|c|c|}
\hline $\mathrm{H}$ & -8.75072 & -1.051411 & 3.795849 & $\mathrm{H}$ & -0.134737 & 1.171369 & -3.895165 \\
\hline $\mathrm{H}$ & -9.045054 & 4.336473 & -0.525021 & $\mathrm{H}$ & 1.171592 & 0.052345 & -4.120799 \\
\hline $\mathrm{H}$ & -7.740015 & 3.810315 & 0.571946 & $\mathrm{H}$ & 2.928627 & 1.398447 & -3.523395 \\
\hline $\mathrm{H}$ & -7.87381 & 3.461231 & -2.478289 & $\mathrm{H}$ & 0.279125 & 3.435446 & 1.153785 \\
\hline $\mathrm{H}$ & -6.797717 & 4.556746 & -1.564612 & $\mathrm{H}$ & -1.837842 & 3.034818 & 2.030669 \\
\hline $\mathrm{H}$ & -4.684711 & 3.466791 & -2.075181 & $\mathrm{H}$ & -3.45477 & 1.56707 & 3.19607 \\
\hline $\mathrm{H}$ & -5.702388 & 2.710324 & -3.335088 & $\mathrm{H}$ & -2.814879 & -0.749199 & 3.810976 \\
\hline $\mathrm{H}$ & -3.661775 & 1.375208 & -2.89122 & $\mathrm{H}$ & 0.377984 & -1.858833 & 4.258747 \\
\hline $\mathrm{H}$ & -3.907773 & 5.110567 & 0.672543 & $\mathrm{H}$ & -1.22137 & -2.464505 & 3.953538 \\
\hline $\mathrm{H}$ & -5.12499 & 0.458757 & -2.494995 & $\mathrm{H}$ & -2.7313 & -2.944519 & 2.713703 \\
\hline $\mathrm{H}$ & -6.636652 & 5.722632 & 3.172972 & $\mathrm{H}$ & -2.773748 & -4.210613 & 1.463468 \\
\hline $\mathrm{H}$ & -4.450066 & 2.769959 & 0.947818 & $\mathrm{H}$ & -3.754041 & -1.434996 & 0.813259 \\
\hline $\mathrm{H}$ & -7.85364 & 1.612221 & 3.289265 & $\mathrm{H}$ & -4.435252 & -2.96178 & 0.21161 \\
\hline $\mathrm{H}$ & -6.194876 & -0.115708 & 2.391765 & $\mathrm{H}$ & -5.254655 & -4.197297 & 2.908074 \\
\hline $\mathrm{H}$ & -4.91757 & 0.444207 & 1.286596 & $\mathrm{H}$ & -6.14762 & -3.913726 & 1.390899 \\
\hline $\mathrm{H}$ & -7.828468 & 0.394739 & 0.834791 & $\mathrm{H}$ & -2.932398 & -3.732016 & -2.54237 \\
\hline $\mathrm{H}$ & -6.776786 & 1.191908 & -0.175368 & $\mathrm{H}$ & 2.178325 & -3.162991 & -2.113233 \\
\hline $\mathrm{H}$ & -5.70266 & -0.771004 & -0.816344 & $\mathrm{H}$ & 2.227144 & -1.622258 & -3.001186 \\
\hline $\mathrm{H}$ & -6.538623 & -1.66507 & 0.476607 & $\mathrm{H}$ & 3.122654 & -0.515465 & -0.883261 \\
\hline $\mathrm{H}$ & -6.703595 & -2.466209 & 6 & $\mathrm{H}$ & 3.135065 & -2.122745 & -0.115266 \\
\hline $\mathbf{H}$ & -7.601208 & -3.897015 & 3.455985 & $\mathrm{H}$ & 4.166809 & -1.142627 & -3.462338 \\
\hline $\mathrm{H}$ & -8.30527 & -3.004988 & -0.194576 & $\mathrm{H}$ & 5.094483 & -0.075691 & -2.397957 \\
\hline $\mathrm{H}$ & -10.297927 & -3.452717 & -1.648878 & $\mathrm{H}$ & 6.435801 & -1.193801 & -4.19534 \\
\hline $\mathrm{H}$ & -9.620181 & 0.232038 & -3.71443 & $\mathrm{H}$ & 6.039944 & -2.782464 & -3.484389 \\
\hline $\mathrm{H}$ & -7.641779 & 0.687496 & -2.284248 & $\mathrm{H}$ & 7.456666 & 0.921864 & -2.198493 \\
\hline$H$ & -2.7702 & -0.388873 & -1.25481 & $\mathrm{H}$ & 8.475644 & -2.944405 & -3.327033 \\
\hline $\mathrm{H}$ & -2.740274 & 0.310515 & 0.381907 & $\mathrm{H}$ & 8.716028 & -1.230009 & -3.764947 \\
\hline $\mathrm{H}$ & -1.649971 & 1.70527 & -2.114153 & $\mathrm{H}$ & 9.498842 & -2.599364 & -1.129784 \\
\hline $\mathrm{H}$ & -1.757587 & 2.406594 & -0.476664 & $\mathrm{H}$ & 11.541394 & -0.149443 & -1.162507 \\
\hline $\mathrm{H}$ & 2.005631 & -3.264189 & 3.480114 & $\mathrm{H}$ & 10.839877 & -1.033121 & 0.220105 \\
\hline $\mathrm{H}$ & 3.004711 & -4.672383 & 1.703567 & $\mathrm{H}$ & 10.5725 & -1.946964 & -2.393566 \\
\hline $\mathrm{H}$ & 1.776629 & -5.032802 & -0.424623 & $\mathrm{H}$ & 10.043383 & 1.825091 & -0.508637 \\
\hline$\Pi$ & -1.613811 & -4.59536 & -0.882754 & $\mathrm{H}$ & 11.376584 & 1.431938 & 0.613538 \\
\hline $\mathrm{H}$ & -0.104789 & -4.757535 & -1.749744 & $\mathrm{H}$ & 10.330551 & 2.422594 & 2.331653 \\
\hline $\mathrm{H}$ & -3.525194 & -1.92315 & -4.128438 & $\mathrm{H}$ & 8.83158 & 2.829757 & 1.454086 \\
\hline $\mathrm{H}$ & -1.951798 & -0.036627 & -4.487677 & $\mathrm{H}$ & 8.610628 & 2.602438 & 3.958016 \\
\hline
\end{tabular}




$\begin{array}{rrrr}\mathrm{H} & 9.012355 & 0.874585 & 3.806269 \\ \mathrm{H} & 6.531989 & 2.258306 & 4.740988 \\ \mathrm{H} & 6.699719 & 0.484071 & 4.721108 \\ \mathrm{H} & 4.695684 & 2.036607 & 3.01123 \\ \mathrm{H} & 2.234427 & -0.795896 & 3.817374 \\ \mathrm{H} & 2.196658 & 0.960015 & 3.528002 \\ \mathrm{H} & 4.219779 & 2.638582 & -1.800942 \\ \mathrm{H} & 6.000709 & 3.257867 & 1.104698 \\ \mathrm{H} & 6.824331 & 5.339043 & -0.024619 \\ \mathrm{H} & 8.295579 & 2.98925 & -3.282609 \\ \mathrm{H} & 10.23646 & -5.484553 & 0.491837 \\ \mathrm{H} & 6.41871 & -3.781895 & -0.48828 \\ \mathrm{H} & 9.063344 & -1.730156 & 2.199018 \\ \mathrm{H} & 6.472159 & -1.220103 & 2.043554 \\ \mathrm{H} & 5.670742 & -1.83576 & 0.569776 \\ \mathrm{H} & 7.355328 & -0.664023 & -0.704045 \\ \mathrm{H} & 7.968638 & 0.028568 & 0.672625 \\ \mathrm{H} & 5.80119 & 0.949406 & 1.209349 \\ \mathrm{H} & 5.218176 & 0.329682 & -0.351591 \\ \mathrm{H} & 4.323142 & 1.342867 & 4.609816 \\ \mathrm{H} & 3.634018 & 0.635823 & 1.509166 \\ \mathrm{H} & 3.672928 & -1.115901 & 1.823273 \\ \mathrm{H} & 3.21409 & 3.060594 & 0.421365 \\ \mathrm{H} & 1.569326 & 2.636659 & 2.012721\end{array}$




\section{References}

S1) Tokunaga, Y.; Hayakawa, K.; Miyashita, J.; Kawasaki, T.; Miyagawa, S. Tetrahedron Lett. 2013, 54, 6829-6833.

S2) Grunder, S.; Torres, D. M.; Marquardt, C.; Błaszczyk, A.; Krupke, R.; Mayor, M. Eur. J. Org. Chem. 2011, 478-496.

S3) Burnett, M. N.; Johnson, C. K. Oak Ridge National Laboratory Report ORNL-6895, 1996.

S4) Gaussian 16, Revision A.03, Frisch, M. J.; Trucks, G. W.; Schlegel, H. B.; Scuseria, G. E.; Robb, M. A.; Cheeseman, J. R.; Scalmani, G.; Barone, V.; Petersson, G. A.; Nakatsuji, H.; Li, X.; Caricato, M.; Marenich, A. V.; Bloino, J.; Janesko, B. G.; Gomperts, R.; Mennucci, B.; Hratchian, H. P.; Ortiz, J. V.; Izmaylov, A. F.; Sonnenberg, J. L.; Williams-Young, D.; Ding, F.; Lipparini, F.; Egidi, F.; Goings, J.; Peng, B.; Petrone, A.; Henderson, T.; Ranasinghe, D.; Zakrzewski, V. G.; Gao, J.; Rega, N.; Zheng, G.; Liang, W.; Hada, M.; Ehara, M.; Toyota, K.; Fukuda, R.; Hasegawa, J.; Ishida, M.; Nakajima, T.; Honda, Y.; Kitao, O.; Nakai, H.; Vreven, T.; Throssell, K.; Montgomery, J. A., Jr.; Peralta, J. E.; Ogliaro, F.; Bearpark, M. J.; Heyd, J. J.; Brothers, E. N.; Kudin, K. N.; Staroverov, V. N.; Keith, T. A.; Kobayashi, R.; Normand, J.; Raghavachari, K.; Rendell, A. P.; Burant, J. C.; Iyengar, S. S.; Tomasi, J.; Cossi, M.; Millam, J. M.; Klene, M.; Adamo, C.; Cammi, R.; Ochterski, J. W.; Martin, R. L.; Morokuma, K.; Farkas, O.; Foresman, J. B.; Fox, D. J. Gaussian, Inc., Wallingford CT, 2016. 


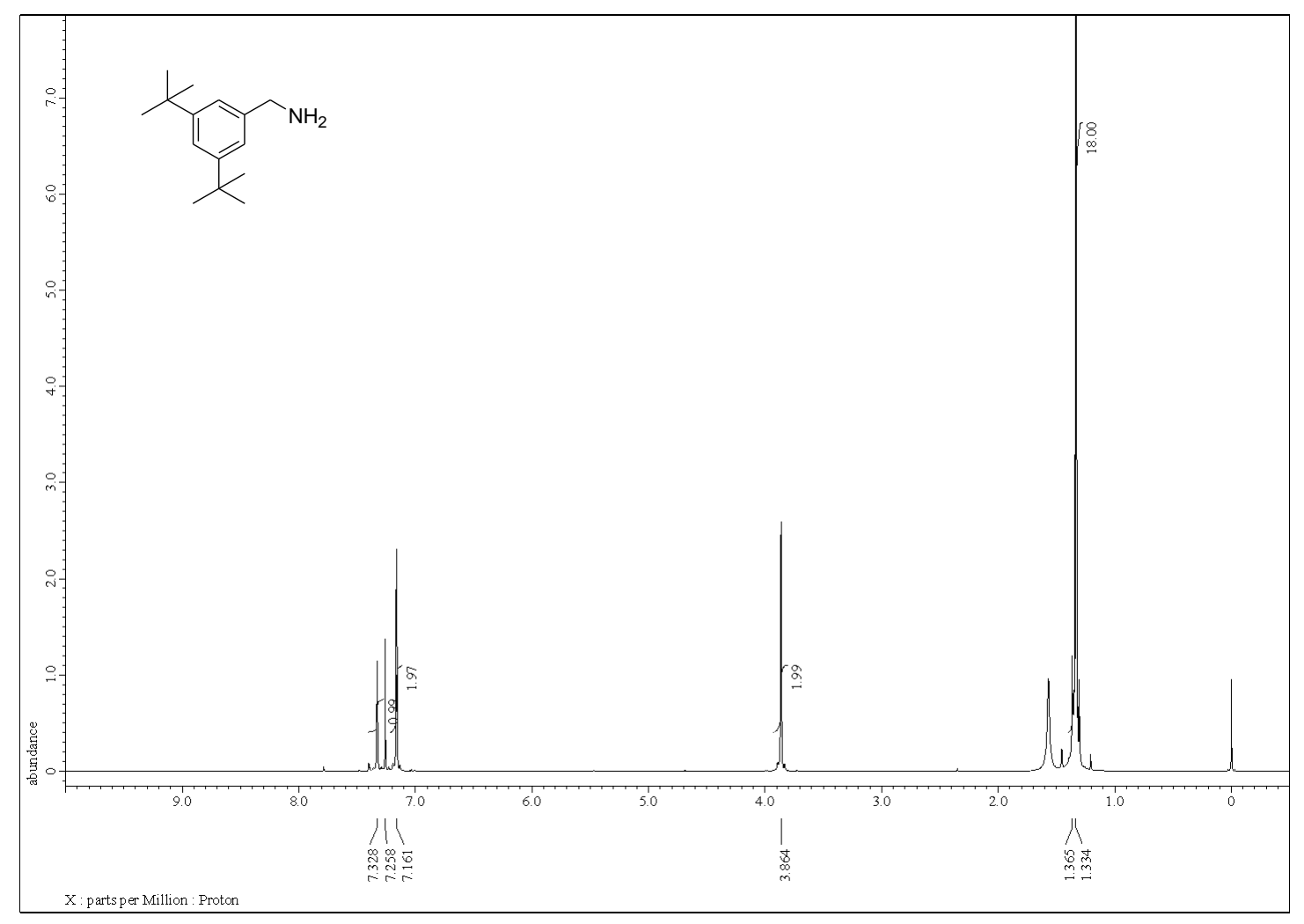

Figure S12. ${ }^{1} \mathrm{H}$ NMR $\left(500 \mathrm{MHz}, \mathrm{CDCl}_{3}\right)$ spectrum of the compound 3. 


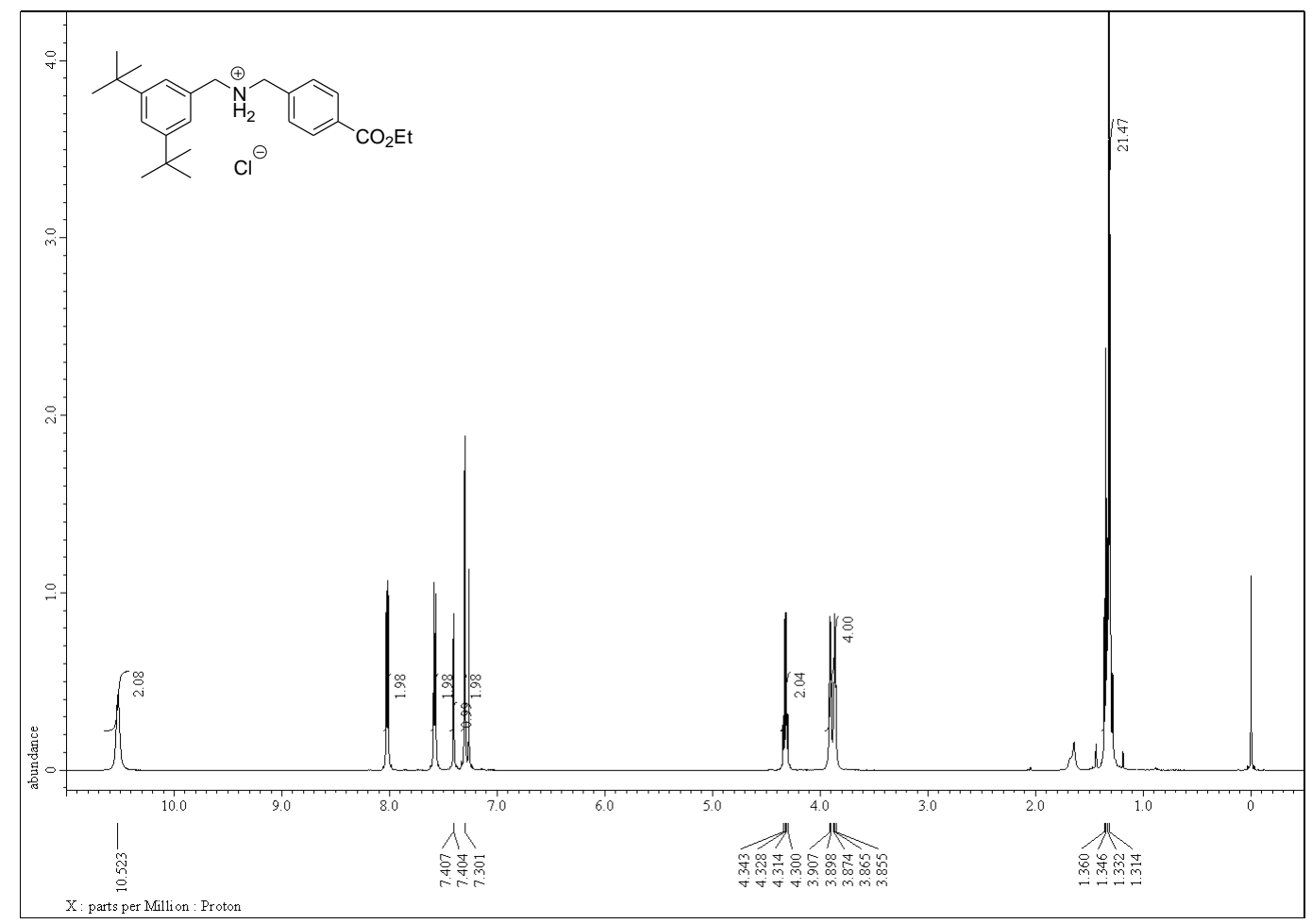

Figure S13a. ${ }^{1} \mathrm{H}$ NMR $\left(500 \mathrm{MHz}, \mathrm{CDCl}_{3}\right)$ spectrum of the compound 4.

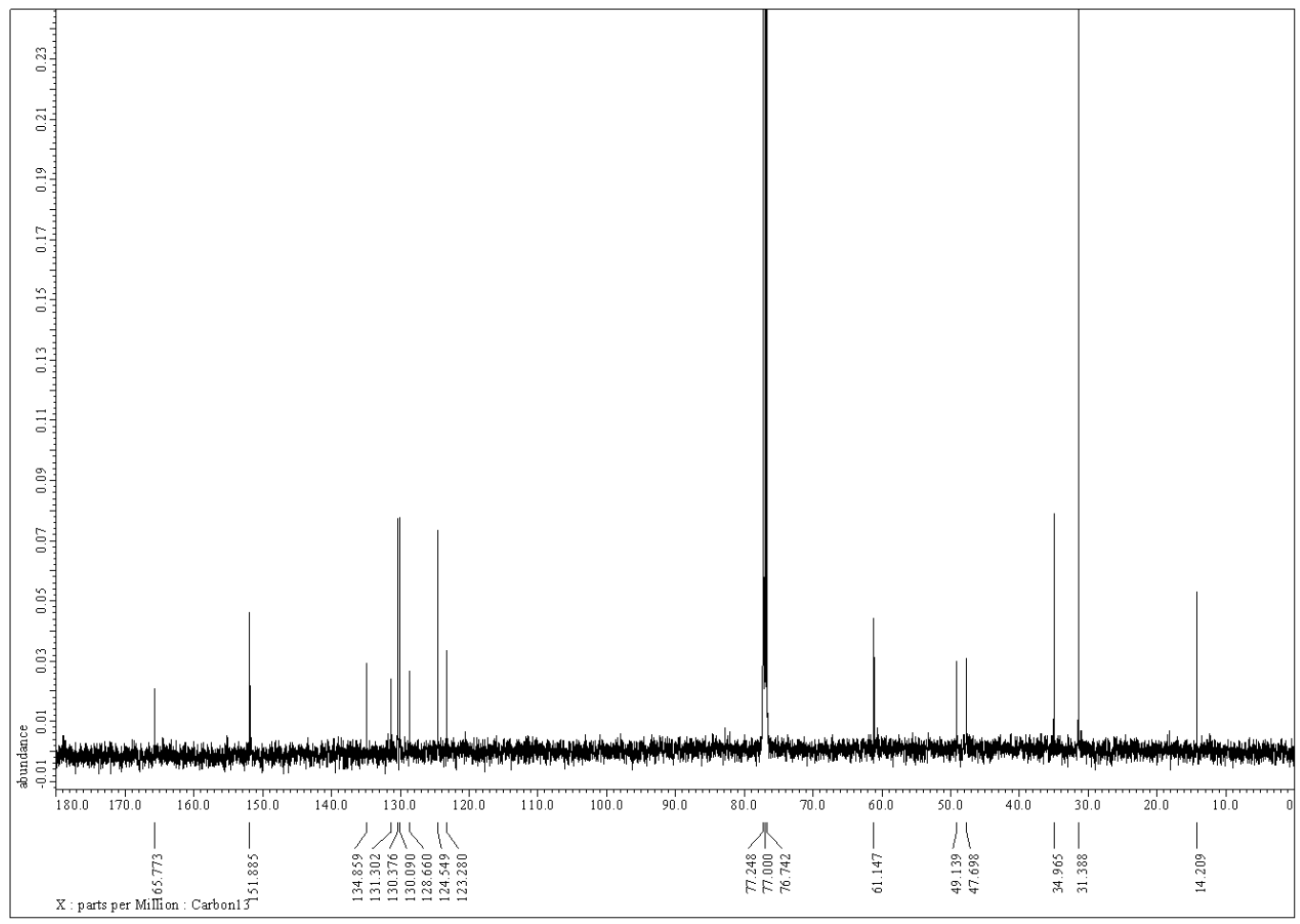

Figure S13b. ${ }^{13} \mathrm{C}$ NMR $\left(125 \mathrm{MHz}, \mathrm{CDCl}_{3}\right)$ spectrum of the compound 4. 


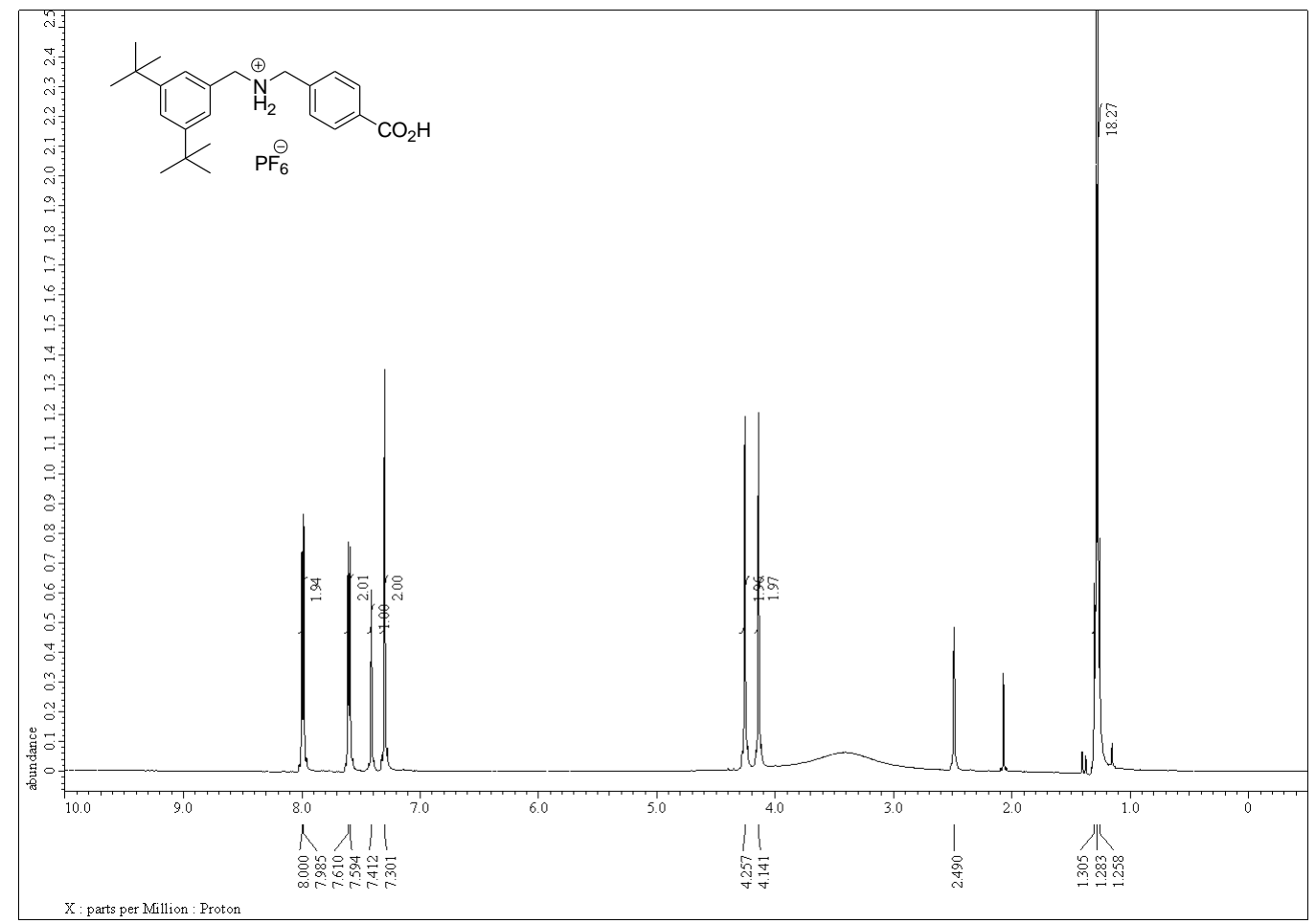

Figure S14a. ${ }^{1} \mathrm{H}$ NMR (500 MHz, DMSO- $\left.d_{6}\right)$ spectrum of the compound 5.

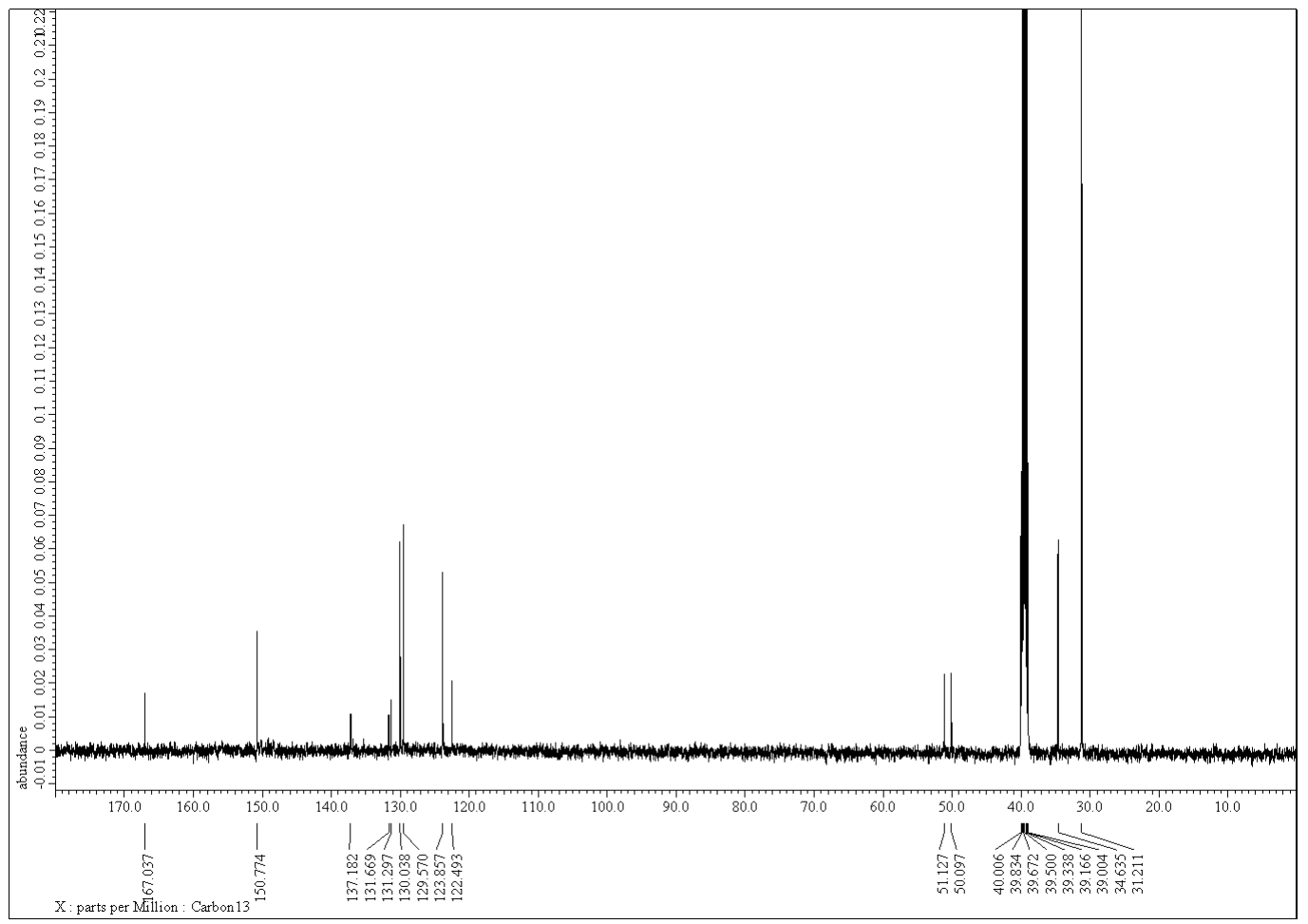

Figure S14b. ${ }^{13} \mathrm{C}$ NMR $\left(125 \mathrm{MHz}, \mathrm{DMSO}-d_{6}\right)$ spectrum of the compound 5. 


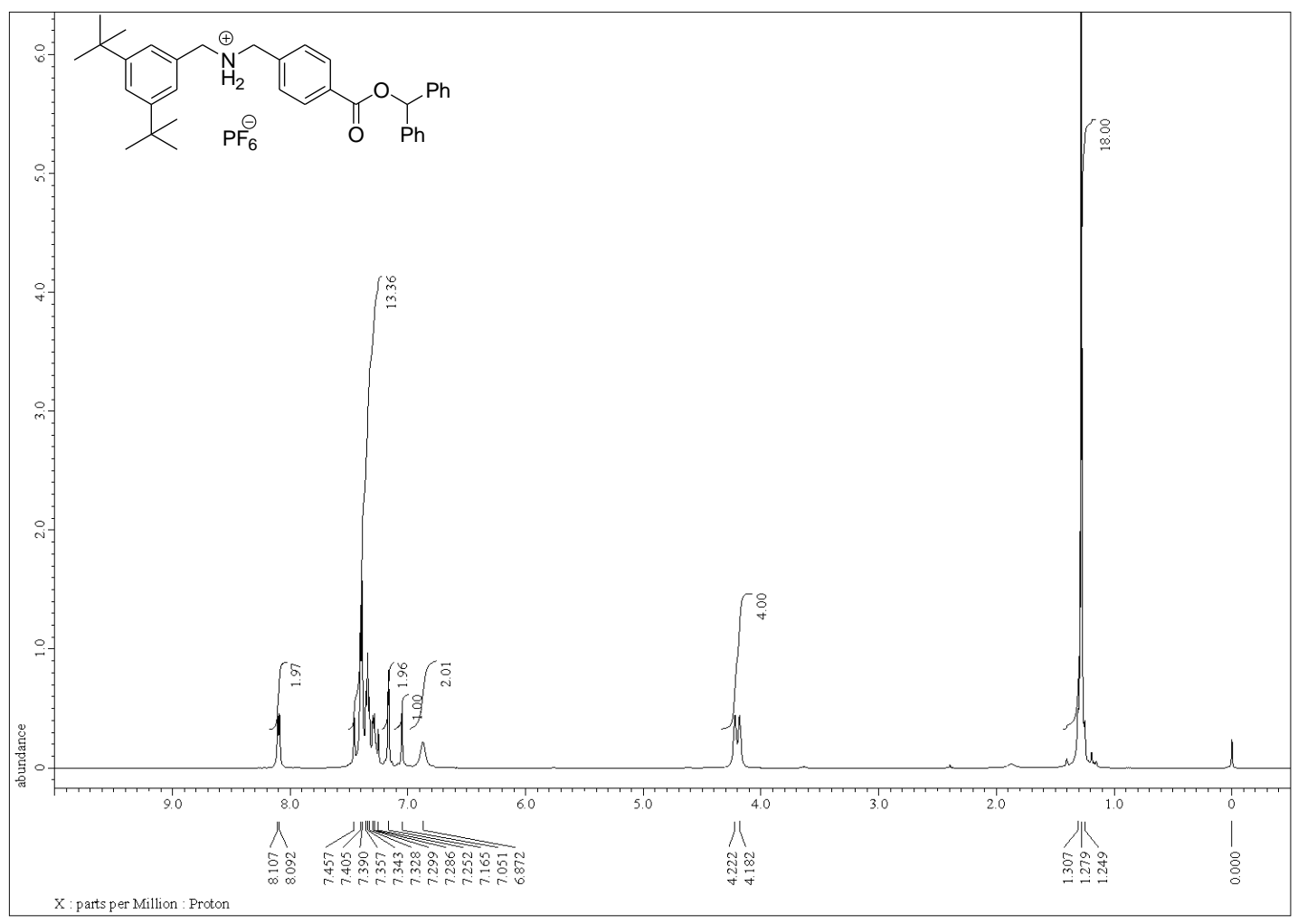

Figure S15a. ${ }^{1} \mathrm{H}$ NMR $\left(500 \mathrm{MHz}, \mathrm{CDCl}_{3}\right)$ spectrum of the compound 6.

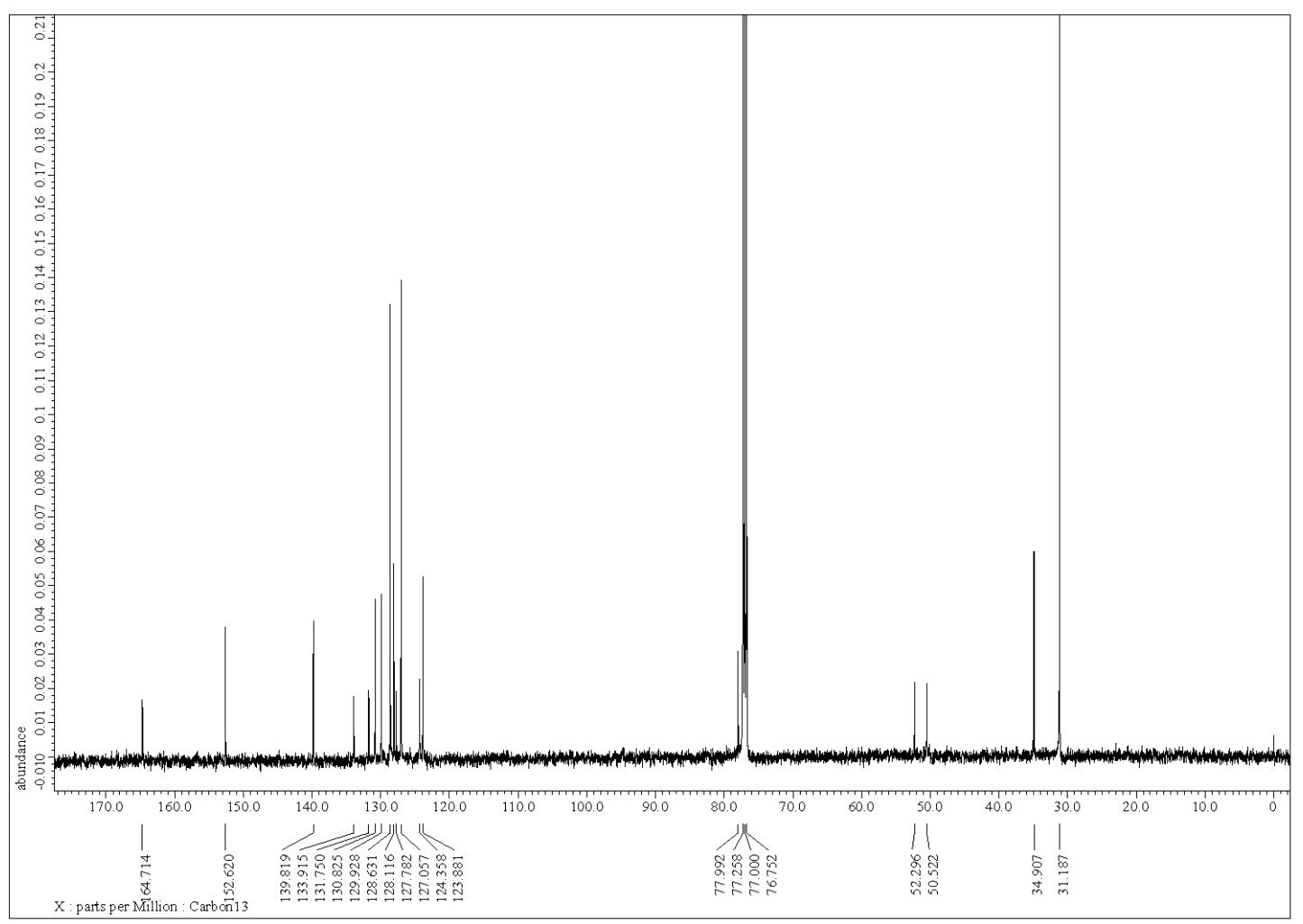

Figure S15b. ${ }^{13} \mathrm{C}$ NMR $\left(125 \mathrm{MHz}, \mathrm{CDCl}_{3}\right)$ spectrum of the compound $\mathbf{6}$. 

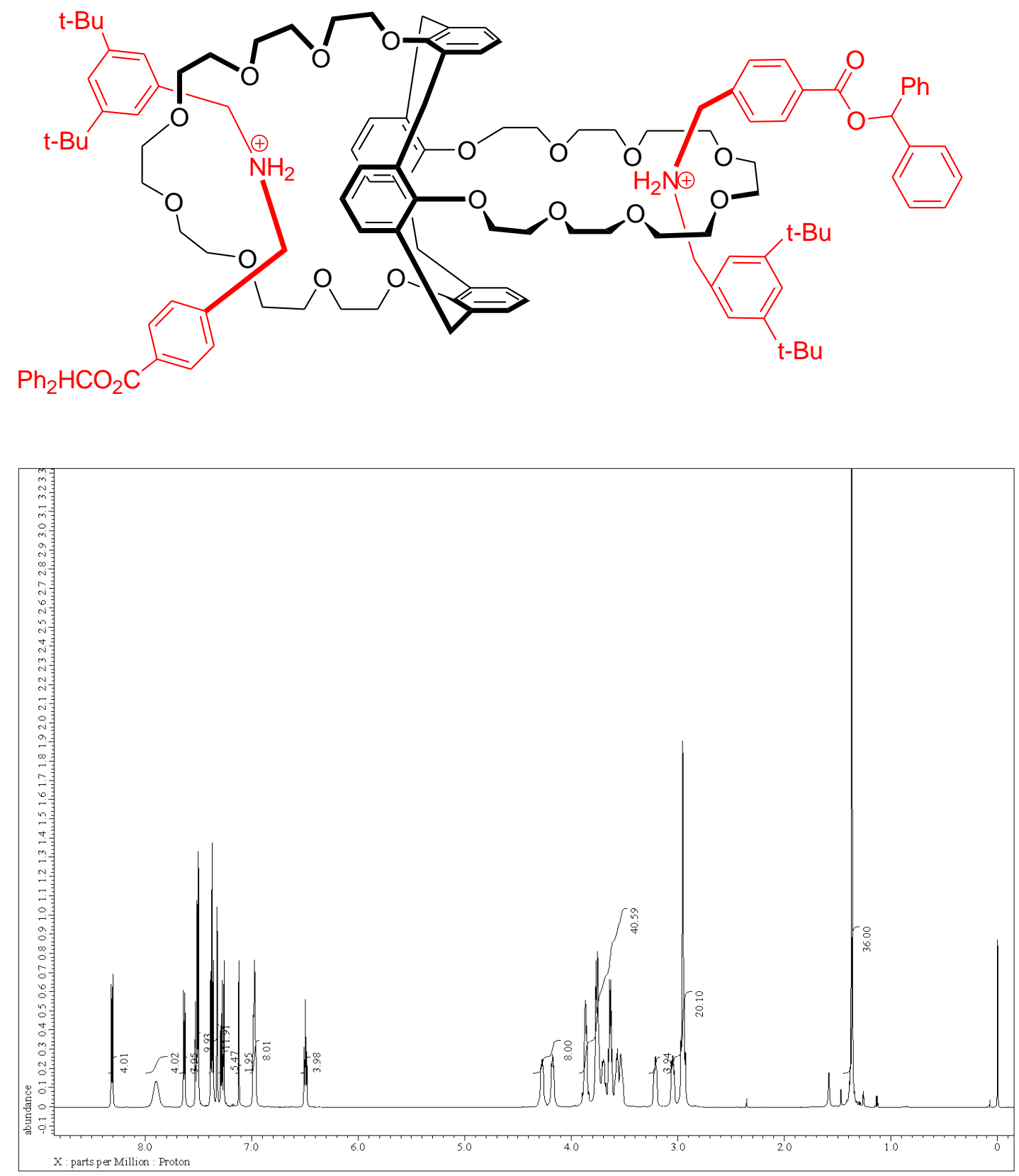

Figure S16a. ${ }^{1} \mathrm{H}$ NMR $\left(600 \mathrm{MHz}, \mathrm{CDCl}_{3}\right)$ spectrum of the [3] rotaxane 1. 


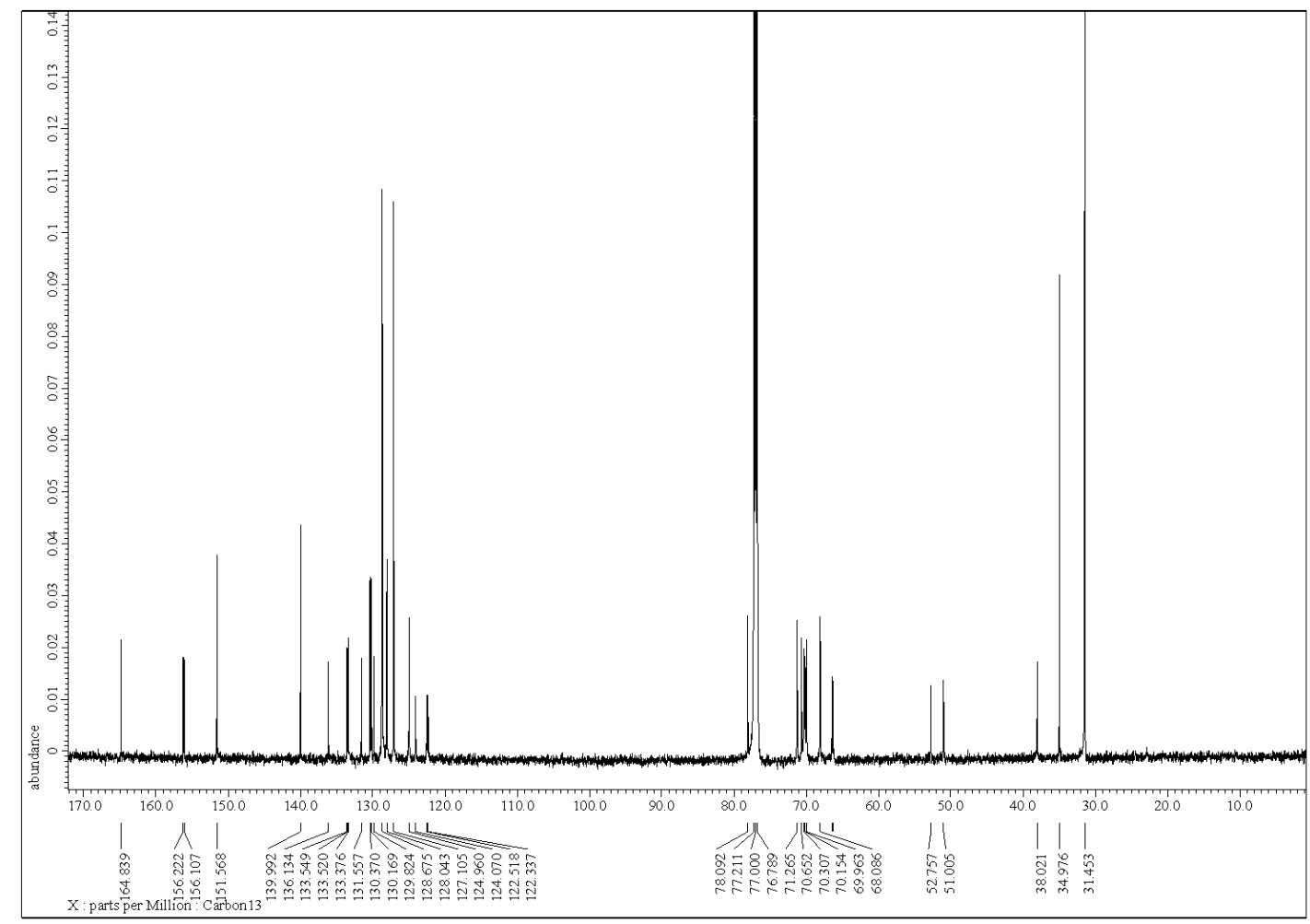

Figure S16b. ${ }^{13} \mathrm{C}$ NMR $\left(150 \mathrm{MHz}, \mathrm{CDCl}_{3}\right)$ spectrum of the [3]rotaxane $\mathbf{1}$. 